\title{
Millimeter-Wave Radio Over Fiber Optical Upconversion Techniques Relying on Link Nonlinearity
}

\author{
Varghese Antony Thomas, Mohammed El-Hajjar, and Lajos Hanzo, Fellow, IEEE
}

\begin{abstract}
A study of advanced upconversion techniques used in radio over fiber (ROF) is provided. With the huge increase in both the number of wireless communication subscribers and the bandwidth required per customer, migrating to higher frequencies, i.e., from lower radio frequency to millimeter-wave carriers, is an essential solution. However, due to the short propagation range of millimeter waves, a large number of radio access points are required for providing reliable coverage, which would increase the infrastructure costs. Hence, the transmission of RF signals between the central (or control) points and radio access points (or remote antenna units) using optical fibers is one of the major access network solutions that have been proposed for future high-bandwidth wireless communication systems. In this paper, we introduce the basics of ROF communication, including optical modulation, the optical channel, and the optical detection techniques. Then we survey the family of advanced optical upconversion techniques that exploit the nonlinearity of the ROF link. Specifically, we describe how optical upconversion can be achieved by exploiting the Mach-Zehnder modulator's nonlinearity, wavelength conversion techniques, or the photodetector's nonlinearity. The wavelength conversion techniques rely on the nonlinearities present in the fiber, in the optical amplifier, or in the electroabsorption modulator.
\end{abstract}

Index Terms-Radio over fiber, direct modulation, external modulation, direct photo-detection, coherent photo-detection, mach zehnder modulator, cross phase modulation, self phase modulation, four wave mixing, stimulated raman scattering, stimulated brillouin scattering, optical frequency modulation, optical phase modulation, optical intensity modulation, optical single side band modulation, optical double side band modulation, optical carrier suppression modulation, sub carrier multiplexing, wavelength division multiplexing, optical $\mathrm{N}$-tupling, optical upconversion, semiconductor optical amplifier, electro-absorption modulator, mach zehnder interferometer, highly nonlinear dispersion shifted fiber, nonlinear optical loop mirror, millimeter wave, 5G, microwave photonics.

\section{INTRODUCTION-WHY RADIO OVER FIBER (ROF)?}

D UE to the huge increase in both the number of wireless communication subscribers and the bandwidth required per customer, migrating to higher frequencies i.e., from radio frequency to millimeter wave carriers is an essential solution to

Manuscript received June 26, 2014; revised February 7, 2015; accepted February 26, 2015. This work was supported by the RC-UK under the auspices of the India-UK Advanced Technology Centre (IU-ATC), the EU's Concerto project, and that of the European Research Council under its Advanced Fellow Grant.

The authors are with the School of Electronics and Computer Science, University of Southampton, Southampton SO17 1BJ, U.K. (e-mail: vat1g10@ ecs.soton.ac.uk; meh@ecs.soton.ac.uk; lh@ecs.soton.ac.uk).

Digital Object Identifier 10.1109/COMST.2015.2409154

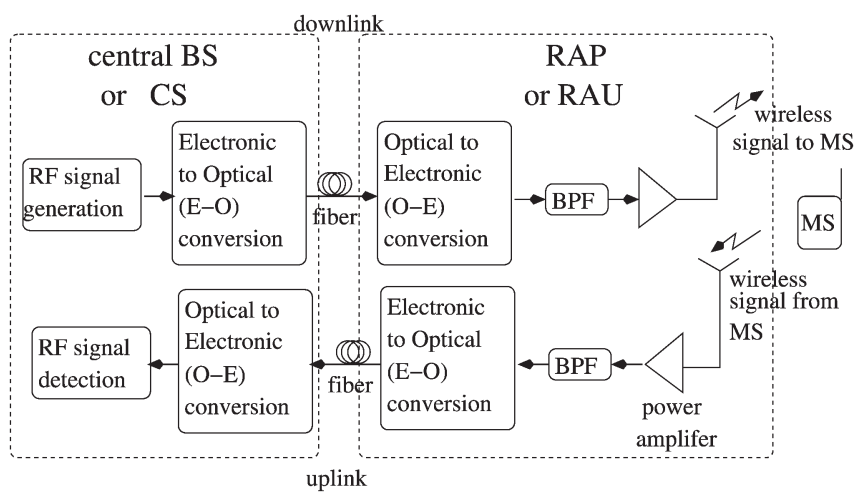

Fig. 1. Simple Radio Over Fiber (ROF) link. (BS-Base Station; CSCentral Station; RAP—Radio Access Point; RAU—Radio Access Unit; BPF— Band Pass Filter).

the overcrowded low frequency spectrum [1]. This migration however imposes two major challenges. Firstly, millimeterwave carriers can provide high bandwidths, but suffer from a low wireless propagation range [2], which would necessitate a large number of Radio Access Points (RAPs). Hence, network operators have to split large cells into smaller cells to meet the increasing demand of bandwidth per user, where increasing the number of Base Stations (BSs) results in an increase in the infrastructure costs [3]. Secondly, the generation of high RF signals using inexpensive mixers would result in significant BER degradation [4].

The transmission of RF signals between the BS and RAPs using optical fibers is one of the major access network solutions proposed for future high-bandwidth wireless communication systems, which is generally referred to as a Radio Over Fiber (ROF) solution [3], [5]-[8]. The simplified ROF link is illustrated in Fig. 1, where the wireless RF signal is converted into an optical signal using an electronic-to-optical (EO) converter that is located at the BS of Fig. 1 [9]. The optical signal then propagates through the fiber and it is detected using an optical-to-electronic (OE) converter that is located in the RAP for recovering the original RF signal. As shown in Fig. 1, the recovered RF signal is amplified, followed by its wireless transmission from the RAP to the Mobile Station (MS). Note that, as shown in Fig. 1, some of the literature refers to the BSRAP link [10]-[12] as the Central Station (CS)-Radio Access Unit (RAU) link ${ }^{1}$ [13], [14]. Both terminologies are valid and used in the literature interchangeably. In this paper, we opted for

\footnotetext{
${ }^{1}$ Many thanks to the anonymous reviewer for pointing this out.
} 


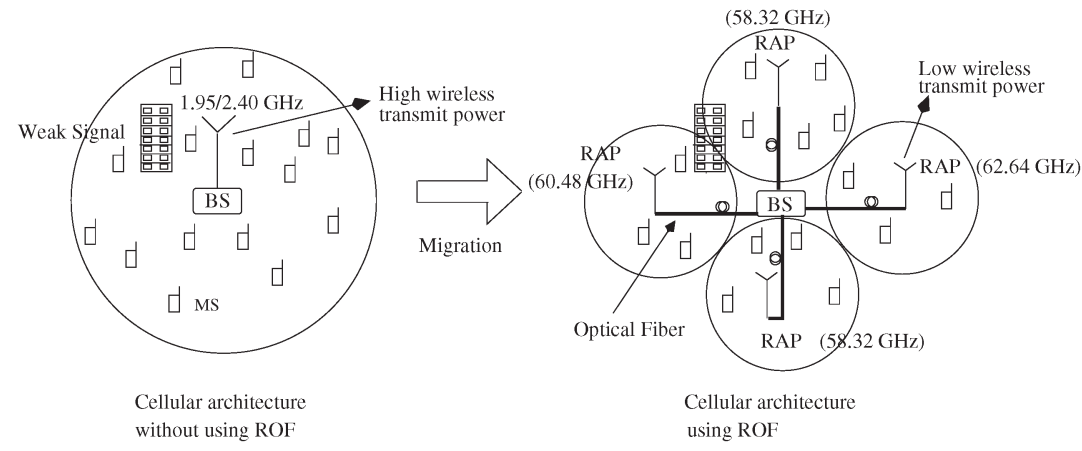

Fig. 2. Cellular architecture.

using the BS-RAP terminology [10]-[12]. The important point to note here is that the ROF link exists between a point that performs centralized signal processing and a low complexity antenna-bearing point.

The ROF technique has several applications including the transmission of WiMAX [15], WiFi [16], audio and video distribution [17]. It may be employed in cellular communication [18], in satellite communication [19], in data communication within trains [20], for implementing indoor networks [21] as well as in phased array and radar communication [22]. Finally, it may be employed in the transmission of the state-of-the-art millimeter-wave signals [23].

Fig. 2 shows the current macrocell-cell based cellular architecture, while a futuristic ROF-aided Distributed Antenna System (DAS) that employs smaller cells is also shown in Fig. 2. ROF is a hybrid technique that amalgamates the concepts of both optical and wireless communication, as observed from Figs. 1 and 2, where this amalgamation retains the benefits of both communication techniques. In this context, the major advantages of a ROF system can be summarised as follows:

1) The glass optical fiber ${ }^{2}$ has a low attenuation of about $0.2 \mathrm{~dB} / \mathrm{Km}$, which enables fiber-linked antennas for covering a large geographical area, as shown in Fig. 2. Again, the ROF architecture of Fig. 2 is also referred to as a DAS [7].

2) The optical fiber is characterised by large bandwidths, which can be exploited using the suite of techniques presented in this paper.

3) Centralised signal processing at the BS and the ability of the BS to serve a number of RAPs that have a simple architecture, as shown in Fig. 2, makes a cellular system relying on numerous small cells economically feasible [24]. Furthermore, better bandwidth/channel allocation using dynamic algorithms as well as technology and hardware upgrades can be readily implemented, when using centralised signal processing at the BS [25].

4) The fraction of the areas where the MS does not receive the downlink wireless signal with sufficient power is minimized, when employing a ROF-assisted cellular architecture [3].

5) Multiple transmitters and receivers can be used for supporting a single MS in order to increase its throughput

${ }^{2}$ Glass optical fiber refers to optical fibers made from silica.

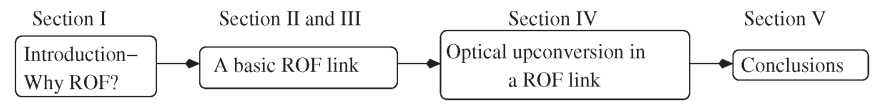

Fig. 3. Organization of the paper.

by exploiting the fading nature of the wireless channel. These techniques are referred to as Multiple Input Multiple Output (MIMO) techniques [26]-[28]. A ROF-aided cellular architecture facilitates the employment of Coordinated Multipoint (CoMP) solutions, where each MS of the ROF-aided cellular architecture of Fig. 2 is served by multiple RAPs [5].

6) The presence of a stronger line of sight link within the smaller cells of the ROF-assisted architecture of Fig. 2 results in a reduced wireless path loss [3], which facilitates a reduced wireless transmit power at the RAPs, hence providing the additional advantage of a reduced inter-cell interference [29].

The ROF technique of Fig. 1 assists in overcoming the challenges of distributing high-RF signals to a large number of small cells, as seen in Fig. 2. However, the challenge of having to employ BER-degrading, high-RF mixers remains unresolved. Fortunately, several techniques that avoid the employment of high-RF mixers by relying on optical generation of the RF signals have been proposed in the literature [30]. These techniques rely on the non-linearities of the optical link and may collectively be referred to as optical upconversion techniques. Fig. 3 illustrates the organization of the rest of this treatise. Through this paper we introduce a non-specialist reader to the basics of ROF communication in Sections II and III. Afterwards, we present a survey as well as briefly introduce the various ROF optical upconversion techniques in Section IV. Finally, we present our conclusions in Section V. It must be pointed out that throughout this paper, $f_{i}$ refer to frequencies, while $\omega_{i}$ refer to the corresponding angular frequency, which are related as follows:

$$
\omega_{i}=2 \pi f_{i} .
$$

\section{ROF COMMUNICATION BASICS}

Fig. 1 shows the block diagram of a typical ROF link [9], which includes the BS, optical fiber and the RAP. As shown in Fig. 1, the ROF link relies on Electronic to Optical (E-O) conversion followed by transmission over the fiber and finally 


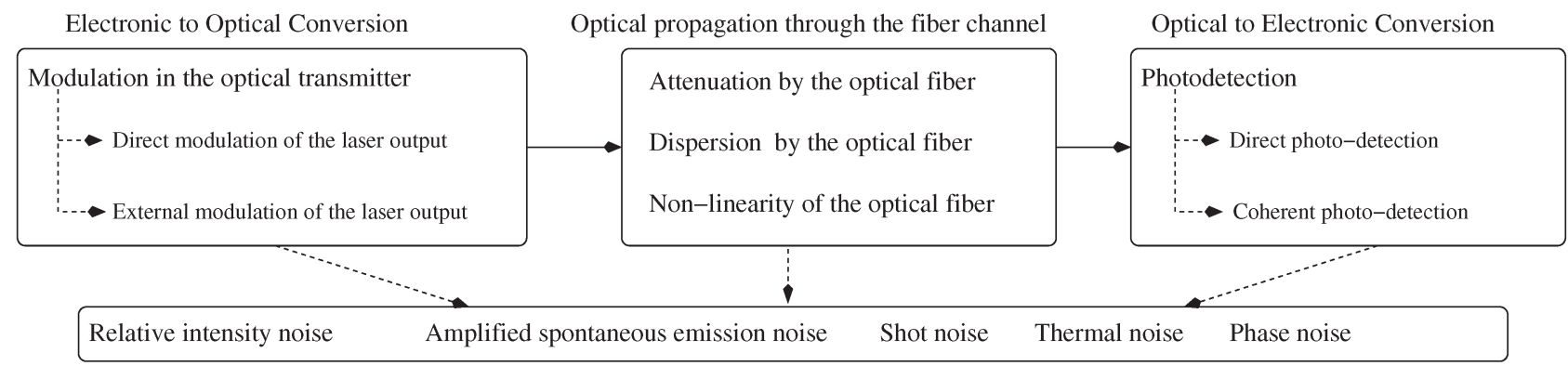

Noise added by the ROF link

Fig. 4. ROF communication basics.

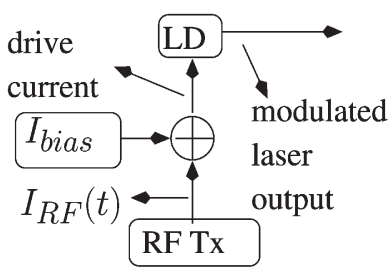

(a)

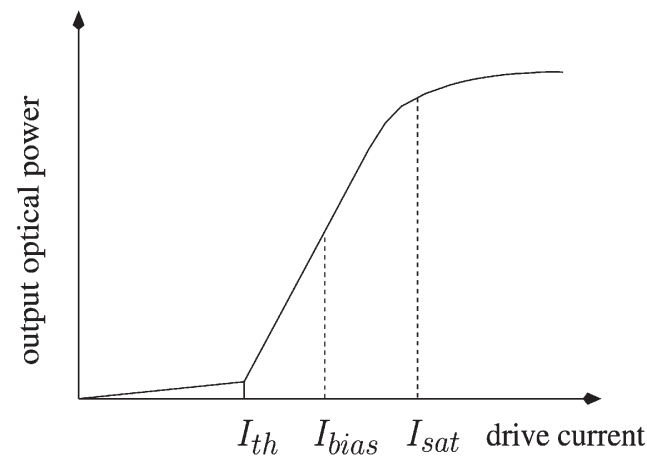

(b)

Fig. 5. Direct modulation (RF Tx-RF Transmitter). (a) Direct modulation setup. (b) Power vs. drive current curve of a laser.

Optical to Electronic (O-E) conversion. Fig. 4 provides an overview of the discussions of Section II. As seen in Fig. 4, E-O conversion involves optical modulation, while the $\mathrm{O}-\mathrm{E}$ conversion includes photo-detection. In what follows, we present a brief overview of the family of optical modulation techniques, of fiber impairments and of photo-detection.

\section{A. Modulation in the Optical Transmitter (E-O Conversion)}

Modulation of the optical carrier can be achieved by directly modulating the optical source or by using an independent optical modulator. Hence, these techniques are referred to as direct modulation and external modulation, respectively. Firstly, we focus our attention on direct modulation of the laser output.

1) Direct Modulation of the Laser Output: In direct modulation, a drive current $I_{\text {drive }}$ is applied to the Laser Diode (LD) in order to generate the optical output. Fig. 5(a) shows the block diagram of direct modulation, where the output optical power of a laser can be inexpensively modulated by applying the output of the RF transmitter as the drive current for the laser. Fig. 5(b) shows the output optical power that can be attained for different drive current values, where this is referred to as the P-I characteristic of the laser. When operating in the linear region of the $\mathrm{P}-\mathrm{I}$ curve, the output power is $P(t)=k\left(I_{\text {drive }}-I_{t h}\right)=$ $k\left(I_{\text {bias }}+I_{R F}(t)-I_{t h}\right)$, where $I_{\text {bias }}$ is the bias current, $I_{t h}$ is the threshold current of the laser and $I_{R F}(t)$ is the modulating $\mathrm{RF}$ signal, while $k$ is the proportionality constant whose value depends on the laser source that is used.

Direct modulation of lasers has multiple limitations [31]. First, as shown in Fig. 6, the laser's direct modulation band-

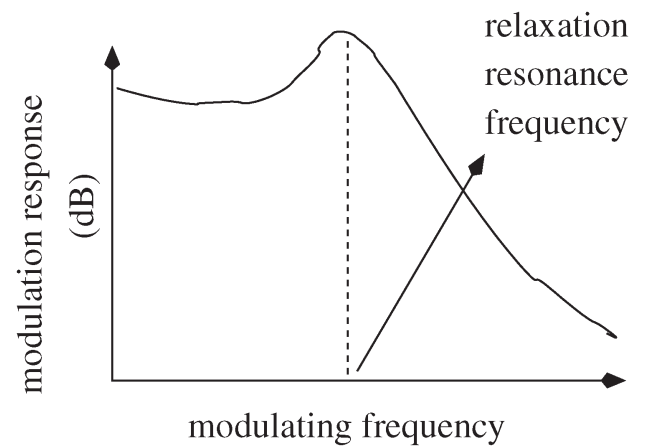

Fig. 6. Modulation bandwidth of the laser.

width limits the frequency range of the employable modulating signals. As seen from Fig. 6, the modulation response peaks at a certain frequency, beyond which the modulation response drops drastically. This frequency limit is called the relaxation resonance frequency and it decides the laser's 3-dB modulation bandwidth. On the other hand, the P-I characteristic of Fig. 5(b) exhibits saturation, which restricts the directly modulated laser's usable output power. Additionally, undesired frequency modulation accompanies the direct modulation of the laser's output intensity. Finally, direct modulation employing signals containing frequencies close to the resonance frequency shown in Fig. 6 results in an increase of both the non-linear products and of the noise in the modulated output [31]. The above limitations are overcome by employing external modulation of the laser.

We now turn out attention to external modulation of the laser output. 


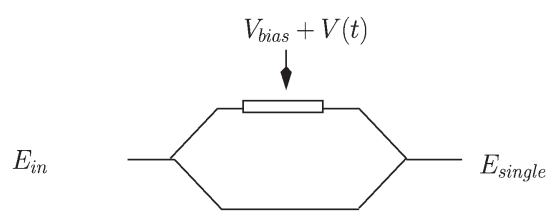

(a)

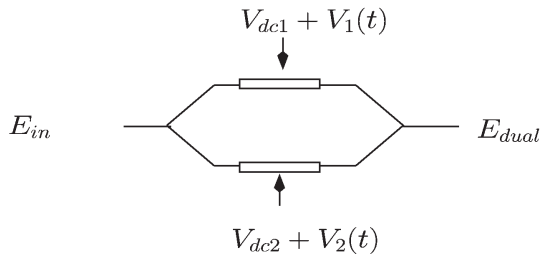

(b)

Fig. 7. Mach-Zehnder Modulator (MZM) external modulators. (a) Single drive MZM. (b) Dual drive MZM.

2) External Modulation of the Laser Output: An optical carrier is a sinusoidal signal, also referred to as a tone, and hence it is characterised by an amplitude, phase and frequency, while the Frobenius norm of the tone is referred to as the intensity. External modulators may rely on modulating both the intensity [32] and the angle, i.e., phase or frequency, [33] of the optical carrier. The commonly employed external intensity modulators include the following.

a) Mach-Zehnder modulator (MZM): In the MZM, the incoming signal is split between its two arms, as shown in Fig. 7 [34]. Either one or both of the arms of the MZM can be driven by a modulating voltage. Hence, they are referred to as singledrive and dual-drive MZMs, as shown in Fig. 7.

In the case of a dual drive MZM, the bias voltage $V_{\text {bias }}$ is applied differentially to the two arms via the voltages $V_{d c 1}$ and $V_{d c 2}$, as shown in Fig. 7, while the modulating voltage $V(t)$ is applied differentially to the two arms via the voltages $V_{1}(t)$ and $V_{2}(t)$. This is represented by: $V_{\text {bias }}=V_{d c 1}-V_{d c 2}$ and $V(t)=V_{1}(t)-V_{2}(t)$. If the output field $E_{i n}=\sqrt{2 P_{i n}} e^{j \omega_{c} t}$ of a laser that operates at an optical power of $P_{i n}$ and at an optical frequency of $f_{c}=\frac{\omega_{c}}{2 \pi} \mathrm{Hz}$ is fed to the MZMs of Fig. 7, then the output optical field $E_{\text {dual }}(t)$ and the intensity $P_{\text {dual }}(t)$ of a dual-drive MZM that is biased at a voltage of $V_{\text {bias }}$ and driven by an electronic signal $V(t)$ can be represented as follows:

$$
\begin{aligned}
E_{\text {dual }}(t) & \frac{1}{2}\left[e^{j\left(\frac{\pi V_{d c 1}}{V_{\pi}}+\frac{\pi V_{1}(t)}{V_{\pi}}\right)}+e^{\left.j\left(\frac{\pi V_{d c 2}}{V_{\pi}}+\frac{\pi V_{2}(t)}{V_{\pi}}\right)\right] \sqrt{2 P_{\text {in }}} e^{j \omega_{c} t}}\right. \\
= & \cos \left(\frac{\pi\left(V_{d c 1}-V_{d c 2}\right)}{2 V_{\pi}}+\frac{\pi\left(V_{1}(t)-V_{2}(t)\right)}{2 V_{\pi}}\right) \\
& \times e^{j\left[\frac{\pi\left(V_{d c 1}+V_{d c 2}\right)}{2 V_{\pi}}+\frac{\pi\left(V_{1}(t)+V_{2}(t)\right)}{2 V_{\pi}}\right] \sqrt{2 P_{i n}} e^{j \omega_{c} t}} \\
= & \cos \left(\frac{\pi V_{\text {bias }}}{2 V_{\pi}}+\frac{\pi V(t)}{2 V_{\pi}}\right) e^{j\left[\frac{\pi\left(V_{d c 1}+V_{d c 2}\right)}{2 V_{\pi}}+\frac{\pi\left(V_{1}(t)+V_{2}(t)\right)}{2 V_{\pi}}\right]} \\
& \times \sqrt{2 P_{\text {in }}} e^{j \omega_{c} t} \\
P_{\text {dual }}(t) & =\left|E_{\text {dual }}(t)\right|^{2} \\
= & P_{\text {in }}\left[1+\cos \left(\frac{\pi V_{\text {bias }}}{V_{\pi}}+\frac{\pi V(t)}{V_{\pi}}\right)\right],
\end{aligned}
$$

where $V_{\pi}$ is the MZM switching voltage. The drive voltage, which changes the phase of the propagating signal by $180^{\circ}$ when applied to the MZM arms, is referred to as the switching voltage. The output optical power of a single-drive MZM that is biased at $V_{\text {bias }}$ and driven by $V(t)$ is also given by Equation (2c).

Fig. 8 shows the MZM's output optical power $P(t)$ for different values of the bias voltage $V_{\text {bias }}$, which is normally referred to as the MZM transmittance. The drive voltage is varied around

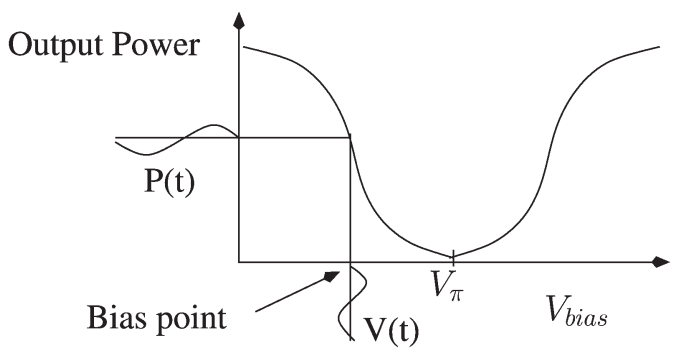

Fig. 8. Mach-Zehnder Modulator (MZM) transmittance.

the bias voltage value in order to achieve optical modulation. Modulation can be interpreted as a voltage fluctuation around the bias point. As seen from Equation (2b), the optical field has exponential terms of the form $e^{j \phi(t)}$, which is the residual phase modulation that is referred to as chirp. This chirp gets converted to intensity modulation after the signal propagates through the fiber, thereby corrupting the data carried by he intensity of the optical carrier. The chirp inflicted by a dual-drive MZM can be varied, where the output would be chirp free, provided we have $V_{1}(t)=-V_{2}(t)=\frac{V(t)}{2}$. This is referred to as the pushpull mode of operation, because the voltages $V_{1}(t)$ and $V_{2}(t)$ are equal and opposite.

On the other hand, the minimum value of $P(t)$ deviates from the ideal value of zero in realistic MZMs, where the extent of deviation is represented by the so-called extinction ratio, $\epsilon=P_{\max } / P_{\min }$, where $P_{\max }$ and $P_{\min }$ are the maximum and minimum values of $P(t)$. Observe from Equation (2c), that the minimum value of $P(t)=0$ occurs, when we have $V_{\text {bias }}=V_{\pi}$ and $V(t)=0$. Thus, the ideal value of extinction ratio is $\infty$. However, non-ideal MZMs have a finite extinction ratio, where the optical field at the output of a non-ideal MZM associated with $\gamma=(\sqrt{\epsilon}-1) /(\sqrt{\epsilon}+1)$ is:

$$
\begin{aligned}
& E_{\text {dual }}(t) \\
& =\frac{1}{2}\left[e^{j\left(\frac{\pi V_{d c 1}}{V_{\pi}}+\frac{\pi V_{1}(t)}{V_{\pi}}\right)}+\gamma e^{j\left(\frac{\pi V_{d c 2}}{V_{\pi}}+\frac{\pi V_{2}(t)}{V_{\pi}}\right)}\right] \sqrt{2 P_{i n}} e^{j \omega_{c} t} .
\end{aligned}
$$

b) Electro-absorption modulator (EAM): The application of an electronic voltage to the EAM changes its absorbtion, ${ }^{3}$ which is referred to as the Franz-Keldysh effect [35]. This effect can be exploited for modulating an electronic signal onto the optical carrier, where the amplitude of the output optical field reduces by a factor of $T(V)$, with $T(V)$ being the

\footnotetext{
${ }^{3}$ The absorption of an optical device refers to the phenomenon of a portion of the propagating optical power being absorbed by the device material.
} 


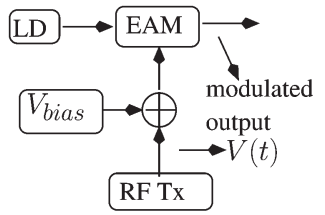

(a)

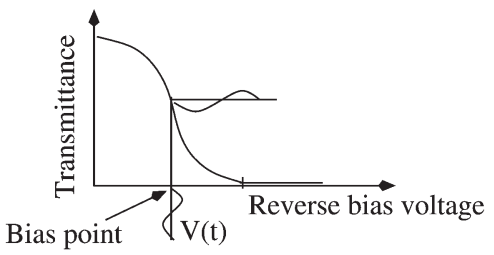

(b)
Fig. 9. External modulation using an Electro-Absorption Modulator (EAM). (a) EAM. (b) EAM T(V) vs. V.

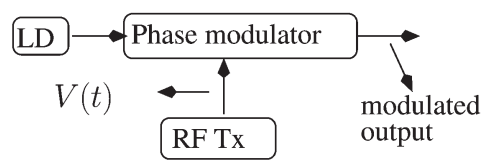

Fig. 10. Optical phase modulator (LD-Laser Diode).

amplitude transmittance, when the reverse bias voltage $V_{\text {bias }}$ applied to the EAM, as shown in Fig. 9(a).

Fig. 9(b) shows the stylized $T(V)$ for different values of the applied reverse bias voltage [36], where $T(V)=0$ corresponds to the MZM output being completely suppressed/extinct, while $T(V)=1$ corresponds to the scenario of no extinction by the EAM. The output optical field $E(t)$ of the EAM modulator in Fig. 9(a) is $E(t) \propto T\left(V_{\text {bias }}+V(t)\right)$, where $V_{\text {bias }}$ is the EAM bias voltage, while $V(t)$ is the modulating drive voltage.

An optical phase modulator is shown in Fig. 10, which is often used to implement external optical phase modulation in ROF links. The phase modulator operates on the same principle as a MZM, except that it has a single arm. The output optical field of a phase modulator, that has a switching voltage of $V_{\pi}$ and is fed by a laser operating at an optical power level of $P_{i n}$, is $E(t)=e^{j \frac{\pi V(t)}{2 V_{\pi}}} \sqrt{2 P_{i n}} e^{j \omega_{c} t}$, where $\omega_{c}$ is the optical carrier frequency and $V(t)$ is the electronic modulating signal.

As shown in Fig. 1, the modulated optical signal then propagates through the optical channel. Hence, we now continue our discussion of a basic ROF link by focusing our attention on the propagation of the optical signal through the fiber channel.

\section{B. Optical Propagation Through the Fiber Channel}

As shown in Fig. 4, the fiber characteristics that affect the propagating optical signal include fiber attenuation, fiber dispersion and fiber non-linearities, as discussed in the following sections.

1) Attenuation of the Optical Fiber: Material absorption and the Rayleigh scattering phenomenon [37] results in the reduction of the signal power, as the signal propagates through the optical fiber. If $P_{\text {transmitted }}$ is the optical power that enters the fiber, while $P_{\text {received }}$ is the optical power after the signal has propagated through $z \mathrm{~km}$ of the fiber, then we have $P_{\text {received }}=$ $P_{\text {transmitted }} e^{-\alpha z}$, where $\alpha$ is the fiber attenuation constant that has a typical value of $0.2 \mathrm{~dB} / \mathrm{Km}$ [37].

2) Dispersion by the Optical Fiber: The fiber's refractive index $n(\omega)$ changes, as the angular frequency $\omega$ of the propagating optical wave changes [37]. Moreover, if $v_{p}$ is the phase velocity of the propagating optical wave, then we have $v_{p}(\omega)=$ $c / n(\omega)$ and hence this dependence of the propagation velocity on the optical frequency results in dispersion. If $\omega_{c}$ and $\lambda_{c}$ are the angular frequency and wavelength of the optical carrier, then the dispersion parameter D can be represented as [37]:

$$
\begin{aligned}
D & =-\frac{2 \pi c}{\lambda_{c}^{2}} \beta_{2} \\
\text { where } \beta_{2} & =\left[\frac{1}{c}\left(2 \frac{d n}{d \omega}+\omega \frac{d^{2} n}{d \omega^{2}}\right)\right]_{\omega=\omega_{c}} .
\end{aligned}
$$

This fiber dispersion is also referred to as Group Velocity Dispersion (GVD) or chromatic dispersion [37].

3) Non-Linearity of the Optical Fiber: An electrical insulator that can be polarized by an electric field $\mathbf{E}$ applied to it is referred to as a dielectric medium, such as a fiber-based cable. The total polarization $\mathbf{P}$ responds non-linearly to the electric field $E$ of the light, especially when the optical intensity is high [37], which results in non-linear effects, such as the non-linear variation of the refractive index and the Four Wave Mixing (FWM) phenomena to be detailed below. The total polarization $\mathbf{P}$ is [38]:

$$
\mathbf{P}=\mathbf{P}_{1}+\mathbf{P}_{2}+\mathbf{P}_{3}+\cdots,
$$

where $\mathbf{P}_{\mathbf{i}}$ is proportional to $\chi^{(i)}$ with $\chi^{(j)}$ being the jth order susceptibility tensor having a rank of $j+1$. In Equation (6), the first term is the linear component. By contrast, second-order nonlinear effects like second harmonic generation (SHG) can appear in noncentrosymmetric crystals only ${ }^{4}$ [38]. Glass-based optical fiber does not have significant second-order nonlinear effects, because $\mathrm{SiO}_{2}$ is a symmetric molecule [38]. The previously mentioned effects of SPM/XPM and FWM are rather third order nonlinearities that originate from the nonlinear component $\mathbf{P}_{\mathbf{3}}$ of the polarization, which is proportional to $\chi^{(3)}$ [38]. These effects are rather elastic in the sense that they do not transfer any energy to the crystal. Moreover, these effects can appear both in noncentrosymmetric and in centrosymmetric crystals [38].

The total propagating signal power $P(t)$ affects the value of the fiber's refractive index, which results in a non-linear refractive index effect. Hence, the refractive index varies with time as $P(t)$ varies with time, where the refractive index variation modulates the phase of the optical signal. The fiber's non-linearity induced phase modulations include both SelfPhase Modulation and Cross-Phase Modulation [37], where the propagating signal's own instantaneous power fluctuation versus time results in Self-Phase Modulation (SPM) [37], while Cross-Phase Modulation (XPM) occurs, when multiple optical signals simultaneously propagate through the fiber. In XPM the non-linear distortion suffered by each signal depends not only on its own optical power, but also on the optical power of the co-propagating signals [37].

On the other hand, when multiple optical signals are propagating together through a single optical fiber, the beating of these input waves inside the nonlinear medium generates optical signals at new frequencies, where this is termed as FWM. For example, FWM results in the generation of an

\footnotetext{
${ }^{4}$ The inverted structure of a crystal is obtained by projecting the coordinates which make up the crystal structure all through a single point, so that $(\mathrm{x}, \mathrm{y}, \mathrm{z})$ becomes $(-\mathrm{x},-\mathrm{y},-\mathrm{z})$. In the event of the original structure and inverted structure being identical, the crystal structure is referred to as centrosymmetric, else it is noncentrosymmetric.
} 
optical signal at $f_{F W M}=f_{1} \pm f_{2} \pm f_{3} \mathrm{~Hz}$, when the signals are propagating at optical frequencies of $f_{1}, f_{2}$ and $f_{3} \mathrm{~Hz}$ in the fiber. The most significant FWM frequencies are those at [37]:

$$
f_{F W M}=f_{i}+f_{j}-f_{k},
$$

where, $i, j, k \in 1,2,3$. The above expression includes frequencies of the form

$$
f_{F W M, 1}=2 f_{i}-f_{j} .
$$

Low absolute values of the dispersion parameter $D$ result in enhanced FWM [37].

Furthermore, Stimulated Brillouin Scattering (SBS) and Stimulated Raman Scattering (SRS) [37] constitute a pair of other non-linear effects, where the photons of the incident field get annihilated because the optical power is transferred from a signal at a higher optical frequency to a signal at a lower optical frequency.

Recall from Fig. 1, that the optical signal is converted back to an electronic signal after propagating through the fiber, which is achieved by photo-detection, as shown in Fig. 4. Hence, we continue our discourse by discussing optical photo-detection.

\section{Photo-Detection in the Optical Receiver ( $O-E$ Conversion)}

Photo-detection refers to the process of detecting an optical signal using a photo-diode (PD), which generates a current that is proportional to the power of the incident optical signal. This is typically in the form of square-law photo-detection, where the ratio of the output photo-current to the incident optical power is defined as the responsivity $R$ of the photo-diode. Direct photo-detection [39] and coherent photo-detection are two variations of the photo-detection family, where in direct photo-detection, the photo-current $I(t)$ of an optical field $E_{S}(t)$ is $I(t)=R\left|E_{S}(t)\right|^{2}$ [39]. On the other hand, in coherent photodetection, the received optical field $E_{S}(t)$ is combined with a local oscillator's optical field $E_{l o}(t)$ followed by their joint photo-detection for generating a current $I(t)=R \mid E_{S}(t)+$ $\left.E_{l o}(t)\right|^{2}$. One of the terms obtained by expanding this expression is a product of the two fields, which is referred to as the beat signal. Coherent photo-detection employing a local oscillator's optical frequency $f_{l o}=\frac{\omega_{l o}}{2 \pi}$-which is different from the carrier frequency $f_{s}=\frac{\omega_{s}}{2 \pi}$ of the incident optical signalis referred to as heterodyne photo-detection, where we have $\omega_{\text {diff }}=\omega_{s}-\omega_{l o}$. If $A_{s}$ and $A_{l o}$ are the amplitudes of the received optical signal and the local oscillator signal, respectively, then the heterodyne photo-detection process results in a current $I(t)$ of:

$$
\begin{aligned}
I(t) & =R\left|A_{s} e^{j \omega_{s} t+\phi_{s}}+A_{l o} e^{j \omega_{l o} t+\phi_{l o}}\right|^{2} \\
& =R\left[A_{s}^{2}+A_{l o}^{2}+2\left|A_{s} \| A_{l o}\right| \cos \left(\omega_{d i f f} t+\phi_{s}-\phi_{l o}\right)\right],
\end{aligned}
$$

where $\phi_{s}$ and $\phi_{l o}$ are the phases of the two optical fields. Fig. 12 shows a system in which the baseband/IF signal is modulated onto the $f_{s} \mathrm{~Hz}$ optical carrier. Subsequently, this signal is combined with the $f_{l o} \mathrm{~Hz}$ local oscillator's laser signal, where $\omega_{\text {diff }}=2 \pi f_{r f}$. Afterwards, the combined signal is heterodyne photo-detected, which results in the beat signal being a $f_{r f} \mathrm{~Hz}$ upconverted version of the baseband/IF signal.
Having discussed a basic noise-free ROF link, in the next section we now study the various noise processes that contaminate a realistic ROF link.

\section{Noise Added by the ROF Link}

As seen in Fig. 4, the noise imposed on a ROF link includes Relative Intensity Noise (RIN), Amplified Spontaneous Emission (ASE) noise, shot noise and receiver thermal noise. When using a constant drive current, the spontaneous emission of photons results in a laser exhibiting certain fluctuations in the output frequency, phase as well as the intensity [37], which is the RIN. The SNR is degraded by the intensity noise, while a non-zero spectral linewidth around the output frequency arises from the phase/frequency noise [37]. When considering an average photo-current of $I_{d c}$, then the RIN noise power can be expressed as [37]:

$$
\sigma_{R I N}^{2}=K_{R I N} I_{d c} \Delta f
$$

where $K_{R I N}$ is a device-dependent value that is usually expressed in $\mathrm{dB} / \mathrm{Hz}$ and $\Delta f$ is the bandwidth over which the noise power is measured in the receiver. A typical value of $K_{R I N}$ is $-150 \mathrm{~dB} / \mathrm{Hz}$. Additionally, the ASE noise is generated by the spontaneous emission within an optical amplifier and then amplified by the amplifier's gain mechanism [37]. Furthermore, the fact that the optical field and the electric current consists of discrete entities called photons and electrons results in a quantum noise effect in the photo-detected signal, which is called Shot Noise [37]. If $e$ is the charge of an electron, then the shot noise power can be expressed as [37]:

$$
\sigma_{\text {shot }}^{2}=2 e I_{d c} \Delta f \text {. }
$$

The temperature-dependent random motion of the electrons results in the photo-detected signal containing Thermal Noise that is added by the load resistor and the electrical amplifiers in the optical receiver [37].

Finally, the laser's phase noise acts as a dominant limiting factor in coherent optical links, where a carrier recovery circuit may have difficulties in tracking rapid phase variations, thereby resulting in performance degradation [23]. The effects of phase noise are characterized by the total linewidth-to-bit rate ratio [37]. Here the total linewidth is the sum of the linewidths of the transmitter laser and the local oscillator laser. Note that practical lasers have a finite spectral width around the operating frequency $\omega$, as seen in Fig. 12, where this is referred to as the laser's linewidth.

While optical modulation may be achieved through direct or external modulation of the laser output, as seen in Fig. 4, the specific optical modulation format used has to be tailored to the design of the various ROF links, as seen from the discussions in the next section.

\section{E. Wireless Channel}

The wireless transmission is formulated as follows [26]:

$$
y=H x+n,
$$

where $x$ is the transmitted symbol vector, $n$ is the Gaussian noise vector, while $H$ is the channel matrix and $y$ is the received 


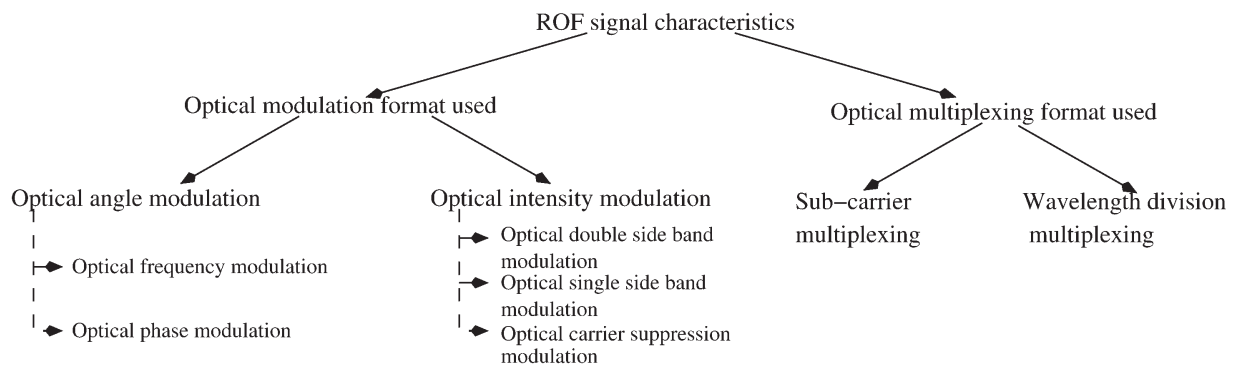

Fig. 11. ROF signal characteristics.

symbol vector. If the wireless channel is modelled as a Gaussian channel, then $H$ is a unitary matrix. On the other hand, $H$ consists of Rayleigh or Rician distributed channel coefficients for a fading channel [26]. An in-depth study of the wireless channel and signalling techniques are available in [26], while this paper's primary focus is on the photonics related issues. At this point it is worth mentioning that the higher wireless pathloss for the case of millimeter wave communications can be overcome by employing beamforming using an array of antenna elements. The outputs of these antennas constructively interfere in the desired direction, while destructively interfering in other directions. A comprehensive study of the millimeter-wave channel and beamforming has been presented in [40] and [41].

In this section, we discuss radio-communication issues related to the integration of a wireless network with a ROF backhaul. This includes power budgeting and the presence of the accumulating noise [42], [43]. These issues are elucidated through the design of a ROF DL that does not employ optical amplification and transmits over a Gaussian wireless channel. The losses and gains in such a link are shown in Figs. 13 and 14. The RF signal $x_{R F}^{B S}(t)$ has a power of $P_{R F}^{B S}$. This power is attenuated by a factor of $L_{o p}$ in the ROF link to produce a photo-detected signal $x_{R F}^{R A P}(t)$ that has a power of $P_{R F}^{R A P}$, where $L_{o p}$ accounts for the $\mathrm{E}-\mathrm{O}$ conversion loss, fiber propagation loss, insertion losses of the optical components as well as the O-E conversion loss. The optical noise power $P_{n}^{o p}$ consists of the RIN, shot noise and thermal noise. In Fig. 13, $x_{R F}^{R A P}(t)$ is corrupted by the optical noise $n_{o p}(t)$ for generating $x_{R F, n o i s y}^{R A P}(t)$. As shown in Figs. 13 and 14, the noise contaminated photo-detected signal is then amplified by $G_{o p}$ and wirelessly transmitted to the MS, thereby resulting in a wireless path loss of $L_{w l}$ or $L_{d b}$ in decibels. In the MS, the wireless link noise $n_{w l}(t)$ of power $P_{n}^{w l}$ is added to the signal to generate $x_{R F, n o i s y}^{M S}(t)$. If the wireless noise power is $k$ times that of the optical noise power, the following can be stated [42]:

$$
\begin{aligned}
S N R_{\text {tot }} & =S N R_{R O F} \frac{1}{1+k \frac{10 \frac{L_{d B}}{10}}{G_{a m p}^{2}}} \\
& =S N R_{R O F} \frac{1}{1+\frac{10^{\frac{L_{d B}}{10}}}{G_{a m p}^{2}}} \text { for } \mathrm{k} \approx 1,
\end{aligned}
$$

where $S N R_{R O F}$ and $S N R_{t o t}$ are the SNR of the ROF link and the total link (optical plus wireless links), respectively.

a) Minimum RAP amplifier gain: Given that $S N R_{R O F}$ is the SNR of the ROF link, the minimum gain $G_{a m p}^{\text {min }}$ required

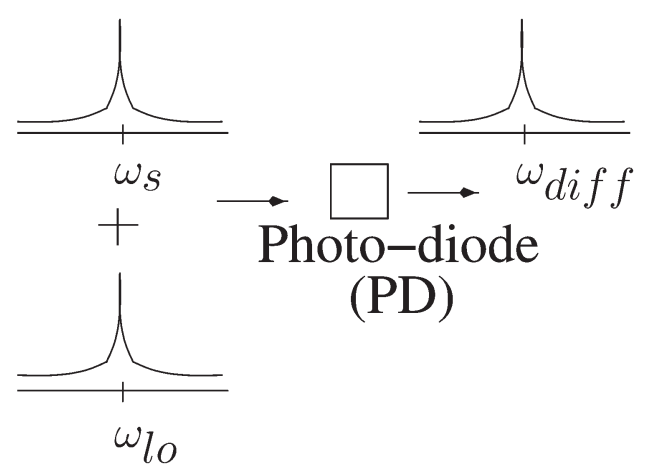

Fig. 12. Heterodyne photo-detection.

of the RAP's electronic amplifier can be calculated using Equation (14) for a scenario of a maximum wireless pathloss of $L_{d B}^{\max }$ at the cell boundary. Note that $S N R_{t o t} \geq S N R_{t o t}^{\text {worst }}$ always has to be satisfied at the MS. Hence we have [42]:

$$
G_{\text {amp }}^{\min }=\sqrt{\frac{\frac{L_{d B}^{\max }}{10}}{\frac{S N R_{R O F}}{S N R_{\text {tor }}^{\text {wort }}-1}}} \text { for } \mathrm{k} \approx 1 .
$$

It can be seen from Equation (15) that the $S N R_{R O F}$ must be higher than the worst-case SNR at the cell boundary [42].

b) Cell size: If a worst-case SNR of $S N R_{\text {tot }}^{\text {worst }}$ is required at the cell boundary, then the maximum tolerable wireless pathloss is [42]:

$$
L_{d B}^{\max }=10 \log \left(\left[\frac{S N R_{R O F}}{S N R_{\text {tot }}^{\text {wor }}}-1\right] G_{\text {amp }}^{2}\right) \text { for } \mathrm{k} \approx 1 .
$$

Observe from Equation (16) that a larger cell having a larger $L_{d B}^{\max }$ needs a higher $S N R_{R O F}$, in other words, a lower fiber length.

Thus, achieving the highest possible $S N R_{R O F}$ is an important design parameter. While limiting the length of the fiber is one way of achieving this, it can also be increased by reducing the $\mathrm{E}-\mathrm{O}$ and $\mathrm{O}-\mathrm{E}$ conversion losses. These losses can be minimized by employing reactive matching circuits instead of resistive matching circuits at the cost of the ROF link's RF bandwidth [42].

\section{ROF SignAl CHARACTERISTICS}

Fig. 11 shows the various attributes of a ROF signal, including the type of optical modulation as well as the type of optical multiplexing format used. As seen in Fig. 11, both the intensity as well as the angle of the optical carrier can be modulated in a ROF link, where the modulating signal is typically an RF signal. 


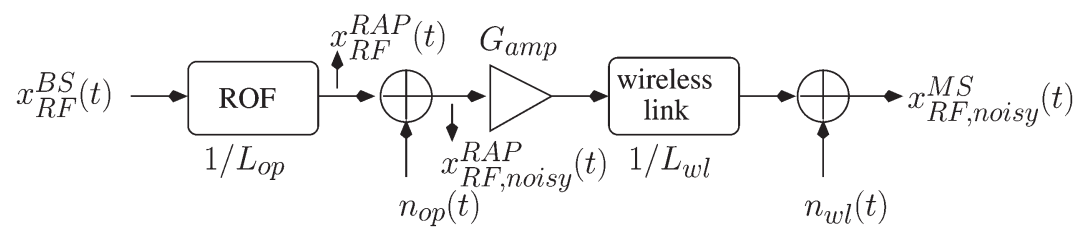

Fig. 13. Losses/gains in ROF DL.

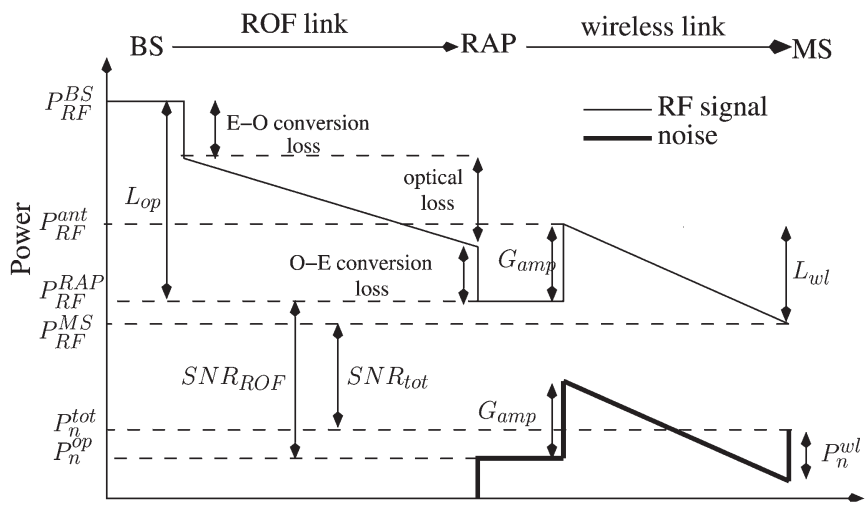

Fig. 14. Power budget.

Optical intensity modulation and optical angle modulation will be described in the following sections. The discussion of the various optical modulation formats is followed by a discourse on the various optical multiplexing formats conceived for ROF communication.

\section{A. Modulation of the Optical Intensity}

The modulation of the intensity ${ }^{5}$ of the optical carrier by a modulating electronic signal is called by definition Intensity Modulation (IM), which may assume the form of Optical Double Side Band (ODSB), Optical Single Side Band (OSSB) or Optical Carrier Suppression (OCS), as discussed in the following sections, as well as shown in Fig. 11.

1) Optical Double Side Band Intensity Modulation: The ODSB signal consists of the lower and upper optical sidebands appearing at both sides of the optical carrier at $f_{c}=\frac{\omega_{c}}{2 \pi} \mathrm{Hz}$, as shown in Fig. 15(a). The carrier-sideband separation seen in Fig. 15(a) equals $\omega_{r f}$ [44], where the modulating RF signal has a center frequency of $f_{r f}=\frac{\omega_{r f}}{2 \pi} \mathrm{Hz}$. An ODSB signal can be generated by both the direct modulation of lasers as well as by external modulation using an EAM [45]. Additionally, an ODSB signal is generated by the single and dual-drive MZMs of Fig. 7, when in Equation (2c) we have $V_{\text {bias }}=V_{\pi} / 2+$ $m 2 V_{\pi}$ or $V_{\text {bias }}=-V_{\pi} / 2+m 2 V_{\pi}$, where $m$ is an integer [32], [46] and $V(t)$ is the RF signal to be transmitted. The bias condition invoked for generating ODSB signals is referred to as quadrature biasing. When employing a dual-drive MZM, a $\pi$ phase difference is maintained between the RF signals $V_{1}(t)$ and $V_{2}(t)$ of Equation (2c) for the sake of generating a chirpfree output [46].

2) Optical Single Side Band Intensity Modulation: An Optical Single Side Band (OSSB) signal contains only one of the

\footnotetext{
${ }^{5}$ The intensity of an optical signal is the square of its amplitude.
}

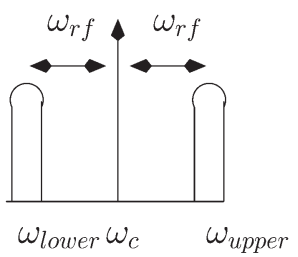

(a)

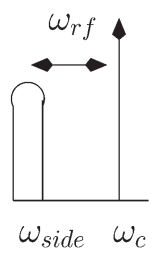

(b)

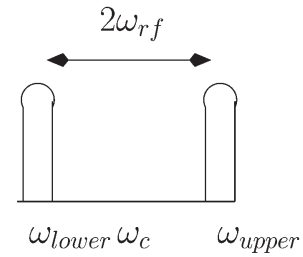

(c)
Fig. 15. Optical modulation formats. (a) Optical Double Side Band (ODSB) signal. (b) Optical Single Side Band (OSSB) signal. (c) Optical Carrier Suppression (OCS) signal.

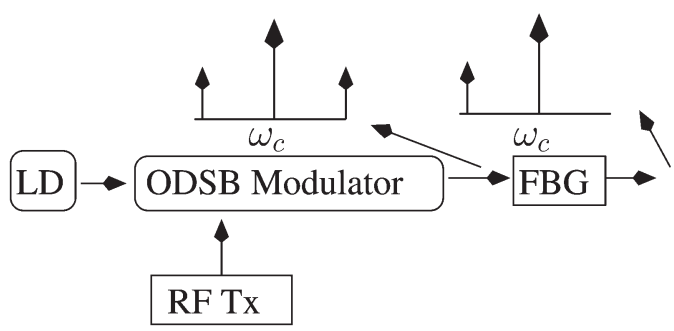

Fig. 16. Optical Single Side Band (OSSB) generation using Fiber Bragg Grating (FBG).

two possible sidebands at $f_{\text {sideband }}=\frac{\omega_{\text {sideband }}}{2 \pi} \mathrm{Hz}$ in addition to the optical carrier at $f_{c}=\frac{\omega_{c}}{2 \pi} \mathrm{Hz}$, as seen from Fig. 15(b). As seen from Fig. 15(b), the carrier sideband separation is $\omega_{r f}$, where the frequency of the modulating RF signal is $f_{r f}=\frac{\omega_{r f}}{2 \pi}$. The two common ways of generating OSSB signals are by using Fiber Bragg Gratings (FBGs) and by using the dual-drive MZM of Fig. 7(b).

Fig. 16 shows how an OSSB signal can be generated using a FBG filter, where one of the sidebands of an ODSB signal can be eliminated using a FBG filter [47]. A FBG is a fiber section produced with a periodic perturbation of the effective refractive index along the length of the fiber core, as shown in Fig. 17(a) [48], where the length of a perturbation period is defined as the grating period [48]. As shown by the transmission profile of Fig. 17(b), a FBG reflects certain wavelengths, while other wavelengths pass through it. The reflected wavelengths, having a frequency of $f_{r}=\frac{\omega_{r}}{2 \pi} \mathrm{Hz}$, is decided by both the periodicity of the perturbation as well as by the effective refractive index of the fiber core. When the RF carrier frequency of the modulating signal is not high, then this technique requires high-selectivity optical filters. Moreover, the refractive index changes with the temperature, thereby resulting in a temperature dependent reflected wavelength value [49]. Additionally, an OSSB signal can be generated by the quadrature biased ${ }^{6}$ dual-drive MZM of

\footnotetext{
${ }^{6}$ In a quadrature biased dual-drive MZM, $V_{\text {bias }}=V_{\pi} / 2+m 2 V_{\pi}$ or $V_{\text {bias }}=-V_{\pi} / 2+m 2 V_{\pi}$ in Equation (2c), where $m$ is an integer.
} 


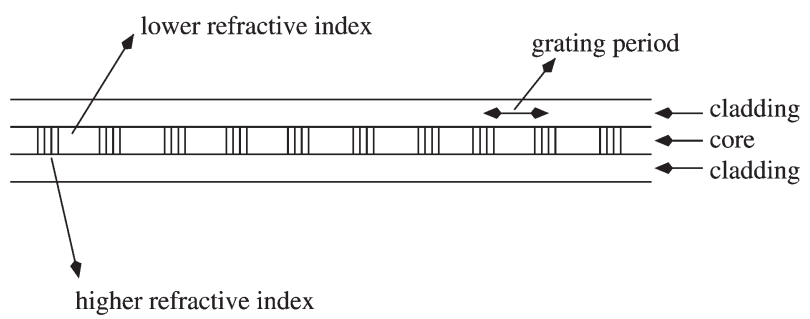

(a)

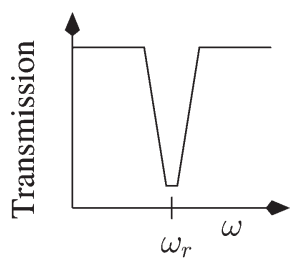

(b)

Fig. 17. FBG. (a) A Fiber Bragg Grating (FBG) filter. (b) Fiber Bragg Grating (FBG) transmission.

Fig. 7(b) by carefully maintaining a phase shift of $\pi / 2$ between the RF signals that are applied to the two arms [34], [46], [50].

3) Optical Carrier Suppression Modulation: An Optical Carrier Suppression (OCS) signal, proposed in [51], consists of two sidebands at $f_{c} \pm f_{r f} \mathrm{~Hz}$, as shown in Fig. 15(c), where the $f_{r f} \mathrm{~Hz}$ is the center frequency of the modulating RF signal. A MZM biased at $V_{\text {bias }}=V_{\pi}+m 2 V_{\pi}$, where $m$ is an integer, generates an OCS signal [30], whose optical sidebands beat during photo-detection, while generating a signal at $2 f_{r f} \mathrm{~Hz}$ [52].

Having completed our discussion of the family of the optical intensity modulation techniques, let us now discuss the family of optical angle-modulation techniques, as shown in Fig. 11.

\section{B. Modulation of the Optical Phase and Frequency (Angle)}

As shown in Fig. 11, optical angle-modulation may involve the modulation of the frequency or phase of the optical carrier. Hence, optical Frequency Modulation (FM) and Phase Modulation (PM) is achieved by modulating either the frequency or phase of the optical carrier, respectively. Both coherent as well as direct photo-detection of optical angle-modulated signals can be implemented. Coherent detection is straightforward, while a prior discriminator aided conversion of the anglemodulated signal to an intensity modulated signal is required for direct detection, where a discriminator characterized by a frequency/phase dependent output intensity is used.

Fig. 18 shows a ROF link that employs a Mach-Zehnder delay Interferometer (MZI), which is the most commonly employed discriminator. In Fig. 18, the phase modulation of the $f_{c}=\frac{\omega_{c}}{2 \pi} \mathrm{Hz}$ output of a laser operating at an optical power of $P_{i n}$ is followed by fiber-based transmission. The anglemodulated signal that enters the RAP is formulated as $E_{i n}(t)=$ $\sqrt{2 P_{i n}} e^{j \omega_{c} t+\phi(t)}$, where the intensity $\left|E_{i n}(t)\right|^{2}$ of the anglemodulated signal is constant, while $\phi(t)$ depends on the modulating RF signal. In the RAP, the incoming angle-modulated signal is divided into two paths and then fed to the arms of the MZI, where the signal in one of the arms experiences a delay of $\tau$ with respect to the other. The output optical field $E_{\text {out }}$ is generated by combining the outputs of the two arms, where the combined output intensity is expressed as [53]:

$$
\begin{aligned}
P_{\text {out }} & =\left|E_{\text {out }}\right|^{2}=\frac{P_{\text {in }}}{2}\left|e^{j \omega_{c} t+\phi(t)}+e^{j \omega_{c}(t-\tau)+\phi(t-\tau)}\right|^{2} \\
& =\frac{P_{\text {in }}}{2}\left[1+1+2 \cos \left(\omega_{c} \tau+\Delta \phi(t)\right)\right],
\end{aligned}
$$

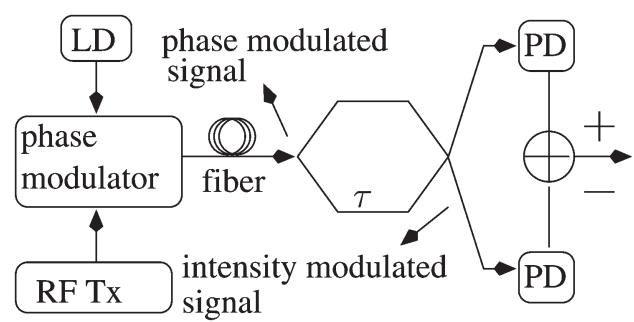

Fig. 18. Phase modulation using Mach-Zehnder delay Interferometer (MZI).

where the MZI is quadrature biased at $\omega_{c} \tau=-\pi / 2$ and $\Delta \phi(t)=\phi(t)-\phi(t-\tau)$. Then, using the small signal approximation of $\sin (\Delta \phi(t)) \approx \Delta \phi(t)$, it can be stated that [53], [54]:

$$
P_{\text {out }}=P_{\text {in }}[1+\sin (\Delta \phi(t))]=P_{\text {in }}[1+\Delta \phi(t)] .
$$

Thus, the angle-modulated optical signal is now converted to an intensity modulated signal, which can now be directly photodetected [53].

Having completed the discussion of the basic optical modulation formats, let us now focus our attention on optical multiplexing formats in the next section.

\section{Optical Multiplexing Techniques}

In order to increase the optical throughput in a multi-user/ multi-RAP system, multiplexing techniques can be employed, including Sub-Carrier Multiplexing (SCM) [55] and Wavelength Division Multiplexing (WDM) [56], as shown in Fig. 11.

1) Sub-Carrier Multiplexing: Frequency multiplexing multiple modulating signals is termed as Sub-Carrier Multiplexing (SCM). For example, as shown in Fig. 19, the pair of signals at $f_{1}=\frac{\omega_{1}}{2 \pi}$ and $f_{2}=\frac{\omega_{2}}{2 \pi} \mathrm{Hz}$ are combined and employed in direct [57] or external modulation [58] of the optical carrier at $f_{c}=\frac{\omega_{c}}{2 \pi} \mathrm{Hz}$. The optical spectrum of the modulating electronic signals as well as of the modulated optical signal is shown in Fig. 19, where the sidebands at $\omega_{c} \pm \omega_{1}$ and $\omega_{c} \pm \omega_{2}$ are the optical channels of the ODSB modulated SCM signal.

Fig. 19 shows an ideal optical spectrum, while the SCM spectrum of Fig. 20 includes the optical harmonics and intermodulation products. Like in Fig. 19, the desired sidebands are at $\omega_{c} \pm \omega_{1}$ and $\omega_{c} \pm \omega_{2}$, when the number of SCM channels is $N=2$. The optical harmonics are generated at $\left(\omega_{c} \pm\right.$ $\left.m \omega_{1}\right)$ and $\left(\omega_{c} \pm m \omega_{2}\right)$ in Fig. 20 with $m$ being an integer, while the optical intermodulation products are located at $\omega_{c} \pm$ $\left(2 \omega_{1}-\omega_{2}\right), \omega_{c} \pm\left(2 \omega_{2}-\omega_{1}\right)$ and $\omega_{c} \pm\left(\omega_{1}+\omega_{2}\right)$. Similarly, 


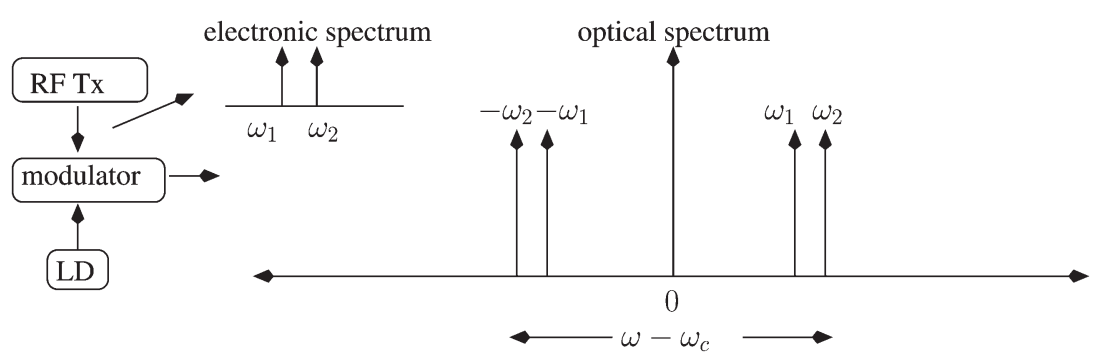

Fig. 19. A Sub-Carrier Multiplexing (SCM) transmitter model.

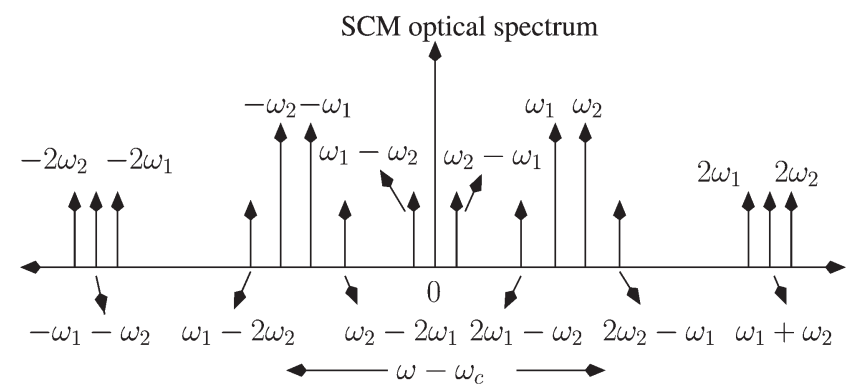

Fig. 20. Sub-Carrier Multiplexing (SCM) optical spectrum.

the photo-detected signal contains harmonics at $m \omega_{1} \mathrm{~Hz}$ and $m \omega_{2} \mathrm{~Hz}$ as well as second and third order intermodulation products at $\left(\omega_{i} \pm \omega_{j}\right)$ and $\left(\omega_{h} \pm \omega_{i}-\omega_{j}\right)$, respectively, where $h, i, j \in\{1,2, . . N\}$ with $h, i$ and $j$ being the SCM channel numbers [59]. It can be seen from Figs. 8 and 9(b) that when an EAM or MZM external modulator is employed, the intensity is non-linearly modulated around the bias point, which produces both upper harmonics and inter-modulation products.

2) Wavelength Division Multiplexing: While only single optical carrier is used in SCM, Wavelength Division Multiplexing (WDM) employs multiple optical carrier frequencies, where electronic signals modulate the optical carrier frequencies to generate sidebands around the carriers, as shown in Fig. 21(a). Afterwards, the modulated optical signals are multiplexed in order to generate the spectrum shown in Fig. 21(a) for the case of three carriers.

WDM based ROF networks employ multiplexers/ demultiplexers and Optical Add Drop Multiplexers (OADMs). A Multiplexer (MUX) and Demultiplexer (DEMUX) pair is used for combining and separating the optical signals at different optical carrier frequencies. Most DEMUXs can operate as a MUX, when operated in the reverse direction, i.e., with a reversal of the input and output ports. As seen in Fig. 21(b), a simple MUX/DEMUX consists of a $1 \times N$ Arrayed Waveguide Grating (AWG) [60], which employs the principle of light-diffraction to separate/combine the wavelengths [60]. In Fig. 21(b), each channel is shown as $\left(\omega_{c, i}, s_{i}\right)$, because it includes both the optical carrier and its sideband, while $\left(\sum \omega_{c, i}, s_{i}\right)$ is used for representing the multiplexed signal. Fig. 21(c) shows the block diagram of an OADM in the RAP, which is used to drop the downlink WDM channel at the optical carrier of say $\omega_{c, 2}$, while simultaneously adding an uplink signal which in this case is at the same optical frequency of $f_{c, 2}=\frac{\omega_{c, 2}}{2 \pi} \mathrm{Hz}$. In Fig. 21(c), the downlink signal at $\omega_{c, 2}$ is shown using a dotted line and is dropped in the OADM and replaced by the uplink signal shown using a bold line.

In the previous two sections of the paper, namely Sections II and III, we introduced the non-specialist reader to the basics of ROF communication as well as discussed the characteristics of a ROF signal. Again, as discussed in Section I, the ROF technique is a solution to the challenge of distributing high-RF signals to a large number of small cells that implements the DAS concept. On the other hand, the challenge of generating these high-RF signals may be addressed through optical generation of the RF signals by optical upconversion. In the next section, we proceed to discuss various optical upconversion techniques that rely on the non-linearities of the ROF link.

\section{OPTICAL UPCONVERSION EXPLOITING ROF LINK NON-LINEARITIES}

The overall Bit Error Rate (BER) achieved by a ROF link that employs high RF frequencies is severely affected by the quality of the broadband mixers. These ROF links involve a stringent cost-performance tradeoff for the case of millimeterwave mixers [4]. This can be overcome by employing optical upconversion techniques, which enable the generation of a photo-detected signal in the RAP at a frequency of $f_{2}=\frac{\omega_{2}}{2 \pi} \mathrm{Hz}$, that is higher than the frequency $f_{1}=\frac{\omega_{1}}{2 \pi}$ of the modulating electronic signal at the BS, as shown in Fig. 23, i.e., we have $f_{2}>f_{1}$. As listed in Fig. 22, optical upconversion can be achieved by using the MZM's non-linearity [30] through architectures like those in Fig. 24, by employing wavelength conversion techniques [61] or by exploiting the photo-detector's non-linearity [62]. The wavelength conversion techniques rely on the inherent non-linearities present in various ROF link components, including the non-linearities in the fiber [61], the optical amplifier [63] or in the EAM [64]. The RF mixers operating at a high frequency significantly degrade the performance of the wireless link. Hence, the major advantage of employing optical upconversion is the ability to avoid the employment of these high frequency RF mixers.

The family of MZM-assisted optical upconversion techniques of Fig. 22 allows us to vary the frequency of the transmitted wireless signal, without having to change the electronic Local Oscillator's (LO's) electronic frequency. Another advantage of MZM-assisted optical upconversion is the ability to generate a WDM signal by using a single external modulator, where each wavelength carries a high RF signal, thereby reducing the system's cost [65]. On the other hand, the family of 


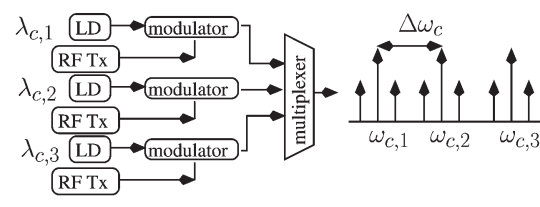

(a)

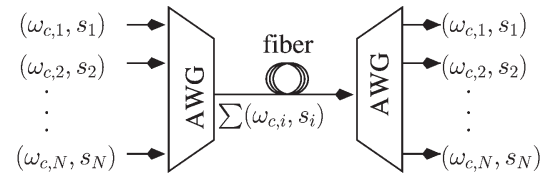

(b)

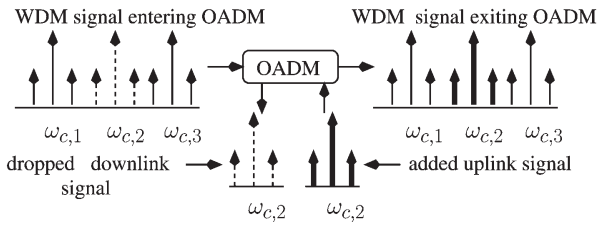

(c)

Fig. 21. Wavelength Division Multiplexing (WDM) (ODSB—Optical Double Side Band; MUX—Multiplexer; DEMUX—Demultiplexer; OADM—Optical Add Drop Multiplexer; AWG-Arrayed Waveguide Grating). (a) An ODSB-WDM transmitter model. (b) A MUX/DEMUX. (c) An OADM.

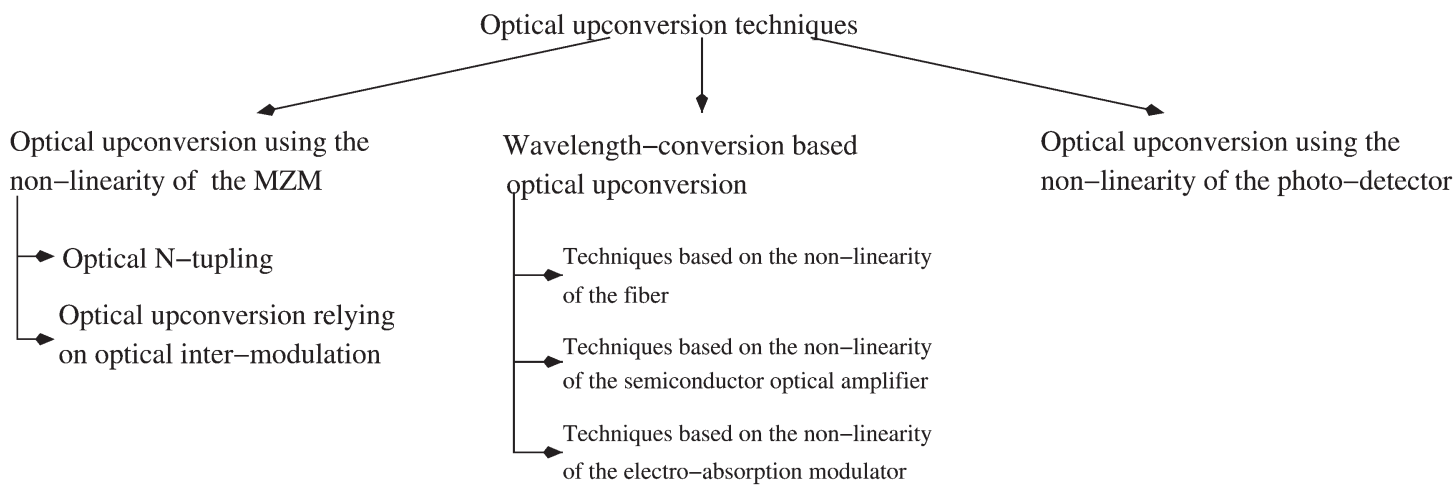

Fig. 22. Optical upconversion techniques.

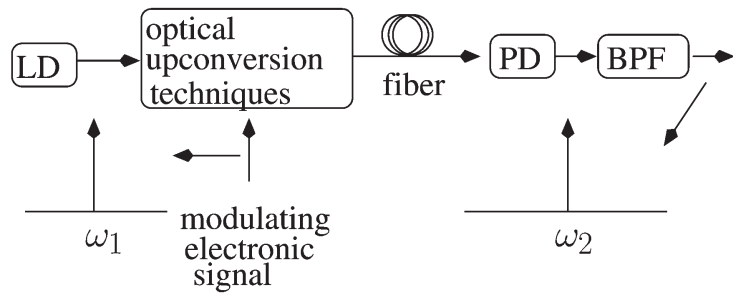

Fig. 23. Optical upconversion (BPF-Band Pass Filter).

wavelength conversion based optical upconversion techniques of Fig. 22 allows optical upconversion to be performed simultaneously with optical switching. For example, the routing of multiple channels at the same wavelength can be achieved by converting the signals to a different wavelength [66].

\section{A. Optical Upconversion Using the Non-Linearity of the MZM}

We commence our discussion of the optical upconversion techniques by considering the family of techniques that rely on the non-linearity of the MZM, as shown in Fig. 22. The non-linear nature of the MZM transmittance, as seen in Fig. 8, results in the generation of harmonics and intermodulation products, where the optical $\mathrm{N}$-tupling technique to be detailed below employs the upper harmonics for achieving optical upconversion. On the other hand, optical intermodulation assisted upconversion exploits the intermodulation products for achieving optical upconversion.

1) Optical N-Tupling: We start our discussion of the family of techniques that rely on the non-linearity of the MZM for optical upconversion by considering the subclass of optical $\mathrm{N}$-tupling techniques shown in Fig. 22. Specifically, the optical modulation techniques that employ an electronic signal at
$f_{L O}=\frac{\omega_{L O}}{2 \pi} \mathrm{Hz}$ for generating a photo-detected signal at the $N^{\text {th }}$ harmonic of $f_{L O}$, are referred to as $\mathrm{N}$-tupling techniques. Optical N-tupling can be achieved using both intensity and angle modulation, where intensity modulation based optical $\mathrm{N}$-tupling is implemented either using single-step or two-step $\mathrm{N}$-tupling. Fig. 24(a) shows the architecture often employed for achieving Single-step N-tupling, where, in the BS, the output of the laser is employed in a single upconversion step achieved by using a MZM that is driven by a RF signal at $f_{L O} \mathrm{~Hz}$. Afterwards, the optical signal is transmitted through the optical fiber and photo-detected in the RAP, where the upconverted photo-detected signal at $f_{R F}=N f_{L O} \mathrm{~Hz}$ can be retained using an electronic filter, as illustrated later in this section [67]. On the other hand, Fig. 24(b) shows the architecture that is employed to achieve Two-step N-tupling, where, in the BS, the output of the laser is employed in the first step, which involved optical modulation using the $f_{I F}=\frac{\omega_{I F}}{2 \pi} \mathrm{Hz}$ baseband/Intermediate Frequency (IF) modulating signal. This is followed by the upconversion step relying on a MZM driven by an electronic LO having a frequency of $f_{L O} \mathrm{~Hz}$. Then, the optical signal is transmitted through the optical fiber to the RAP and photodetected. Subsequently, the upconverted photo-detected signal at $f_{R F} \mathrm{~Hz}$ can be extracted by a filter, ${ }^{7}$ as detailed later in this section [68], where

$$
f_{R F}=N f_{L O}+f_{I F} .
$$

Note that in some cases the upconversion step of the above two techniques might be split into multiple steps. For example, frequency six-tupling might be implemented using frequency doubling followed by frequency quadrupling [67], as detailed later in this section.

\footnotetext{
${ }^{7} N$ is the selected harmonic number.
} 


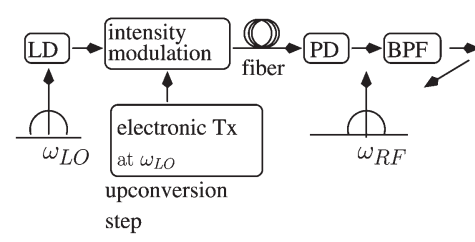

(a)

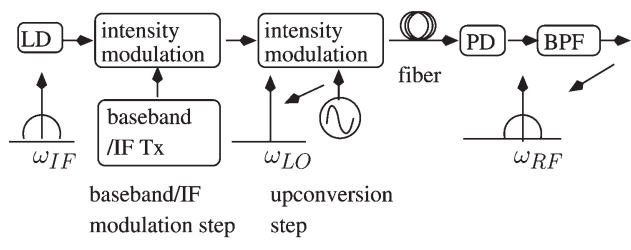

(b)

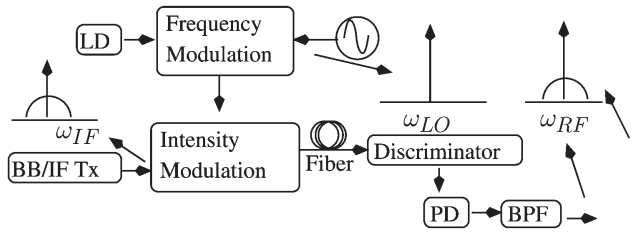

(c)

Fig. 24. N-tupling architectures (BB-Baseband; IF-Intermediate Frequency). (a) One-Step optical upconversion. (b) Two-Step optical upconversion. (c) Optical Frequency Multiplication (OFM).

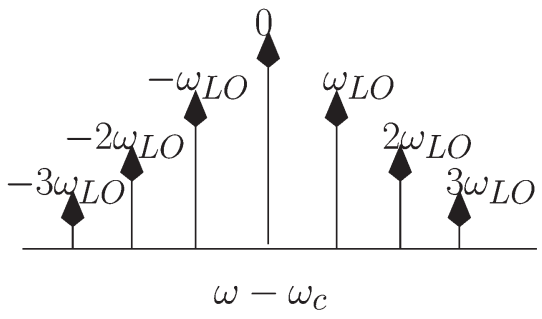

(a)
This carrier is eliminated using

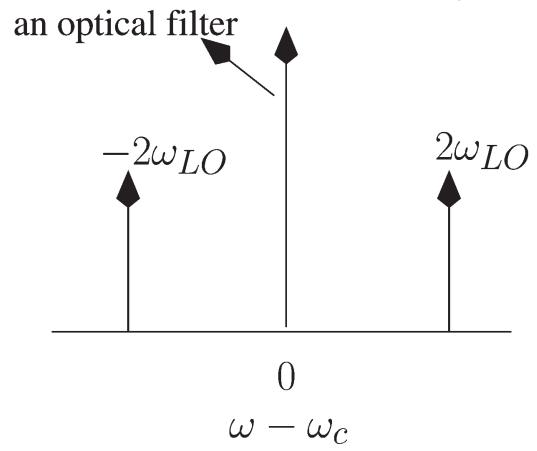

(b)

Fig. 25. N-tupling spectra. (a) Optical Frequency Modulation (OFM). (b) Filtering of optical harmonics.

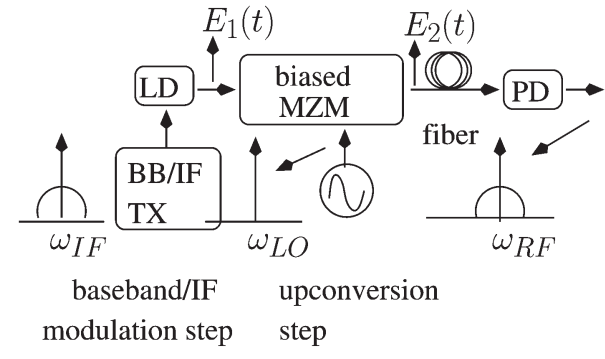

(a)

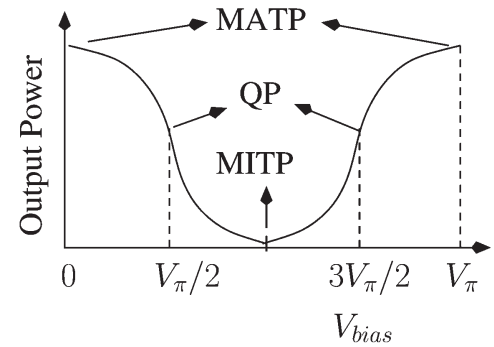

(b)

Fig. 26. MZM assisted N-tupling. (a) MZM assisted N-tupling architecture. (b) MZM bias points.

Another optical N-tupling technique that is robust to dispersion is Optical Frequency Multiplication (OFM) [69]. As shown in Fig. 24(c), instead of generating intensity-modulated harmonics, the output of the laser is frequency/phase modulated using the RF signal, which generates the spectrum of Fig. 25(a), followed by a conversion of these harmonics to intensity modulation using an optical frequency/phase discriminator, as discussed in Section III-B.

In the N-tupling techniques optical filtering may be employed for removing the undesired optical harmonics in order to ensure that the photo-detected signal does not contain harmonics, which are stronger than the one required [70]. For example, if the required photo-detected signal has a frequency of $4 f_{L O} \mathrm{~Hz}$, then it can be generated by the beating of the sidebands located at $\left(\omega_{c} \pm 2 \omega_{L O}\right)$ in Fig. 25(b). However, a strong photo-detected signal is also generated at $2 f_{L O} \mathrm{~Hz}$ by the beating of the optical carrier with each of the sidebands. Hence, the generation of this unwanted signal at $2 f_{L O} \mathrm{~Hz}$ can be avoided by filtering out the optical carrier in the spectrum of Fig. 25(b) prior to photo-detection.
Various MZM biasing techniques can be employed in optical $\mathrm{N}$-tupling, where the point at which a MZM is biased within its non-linear transmittance curve of Fig. 26(b) decides the specific types of harmonics that are generated in the optical and photo-detected signal. Consider the BS of the two step N-tupling architecture of Fig. 26(a) and let $E_{1}(t)$ be the output of the baseband/IF modulation step that is achieved using direct modulation. Then, the upconversion step is implemented using a biased MZM that is driven by the electronic local oscillator signal $V_{L O}(t)=V_{L O} \cos \left(\omega_{L O} t\right)$, as shown in Fig. 26(a). The MZM is usually of dual-drive type because of its ability to operate in the chirp-free push-pull mode. As shown in Fig. 26(b), the MZM can be biased at the Quadrature Point (QP), Maximum Transmission Point (MATP) or Minimum Transmission Point (MITP), as described in the following discussion.

a) Quadrature Point (QP) Biasing: This technique requires the MZM of Fig. 26(a) to be biased at the QP, which corresponds to a bias voltage of $V_{\text {bias }}=\left(V_{\pi} / 2+m 2 V_{\pi}\right)$ or $V_{\text {bias }}=\left(-V_{\pi} / 2+m 2 V_{\pi}\right)$, where $m$ is an integer. The 


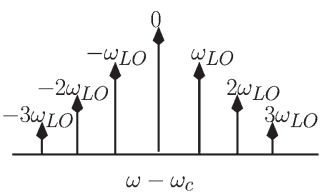

(a)

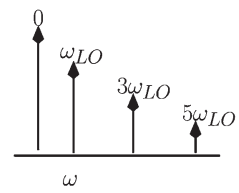

(b)

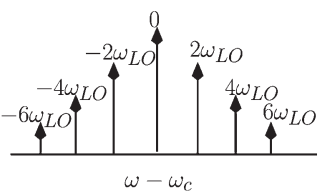

(c)

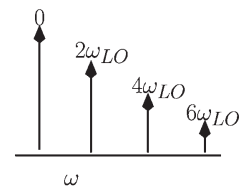

(d)

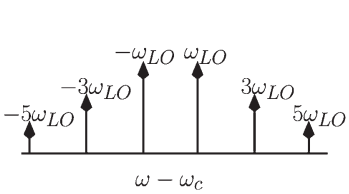

(e)

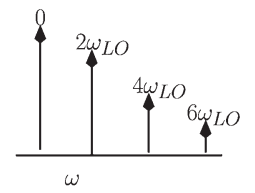

(f)

Fig. 27. Optical and electronic spectrum when employing QP, MATP and MITP biasings (QP-Quadrature Point; MATP-Maximum Transmission Point; MITP_Minimum Transmission Point). (a) QP biased MZM output. (b) PD output for QP biasing). (c) MATP biased MZM output. (d) PD output for MATP biasing. (e) MITP biased MZM output. (f) PD output for MITP biasing.

difference between the two sets of bias points is only in the polarity of the harmonics in the photo-detected signal. If the MZM is of dual-drive type, as shown in Fig. 7(b), then it could be operated in the push-pull mode, as detailed in Section II-A2. Using Equations $(2 b)$ and (2c), the expressions for the optical field $E_{2}(t)$ at the output of the biased MZM in Fig. 26(a) and the photodetected signal $I(t)$ can be formulated as follows:

$$
\begin{aligned}
& E_{2}(t) \\
& =\cos \left( \pm \frac{\pi}{4}+\frac{\pi V_{L O}(t)}{2 V_{\pi}}\right) E_{1}(t) \\
& =\frac{E_{1}(t)}{\sqrt{2}}\left[\cos \left(\frac{\pi V_{L O} \cos \left(\omega_{L O} t\right)}{2 V_{\pi}}\right) \mp \sin \left(\frac{\pi V_{L O} \cos \left(\omega_{L O} t\right)}{2 V_{\pi}}\right)\right] \\
& =\frac{E_{1}(t)}{\sqrt{2}}\left[J_{0}\left(\frac{\pi V_{L O}}{2 V_{\pi}}\right)+2 \sum_{n=1}^{\infty}(-1)^{n} J_{2 n}\left(\frac{\pi V_{L O}}{2 V_{\pi}}\right) \cos \left(2 n \omega_{L O} t\right)\right. \\
& \left.\quad \pm 2 \sum_{n=1}^{\infty}(-1)^{n} J_{2 n-1}\left(\frac{\pi V_{L O}}{2 V_{\pi}}\right) \cos \left((2 n-1) \omega_{L O} t\right)\right]
\end{aligned}
$$

and

$$
\begin{aligned}
& I(t) \propto\left|E_{2}(t)\right|^{2} \\
& =\left[1 \mp \sin \left(\frac{\pi V_{L O} \cos \left(\omega_{L O} t\right)}{V_{\pi}}\right)\right] \frac{\left|E_{1}(t)\right|^{2}}{2} \\
& =\left[\frac{1}{2} \pm \sum_{n=1}^{\infty}(-1)^{n} J_{2 n-1}\left(\frac{\pi V_{L O}}{V_{\pi}}\right) \cos \left((2 n-1) \omega_{L O} t\right)\right]\left|E_{1}(t)\right|^{2}
\end{aligned}
$$

where $J_{n}(x)$ is the Bessel function of order $n$. It can be seen from Equation (20) that the optical signal has both even harmonics, i.e., $N=2 n$, where $n=0,1, .$. and odd harmonics, i.e., $N=2 n-1$, where $n=1,2 .$. , as shown in Fig. 27(a). Fig. 27(a) illustrates the output optical spectrum of the QP biased MZM. Additionally, it can be seen from Equation (21) that the photo-detected signal consists of a signal at the carrier and odd harmonic values associated with $N=2 k-2 \ldots$, as shown in Fig. 27(b). Note that Fig. 27(a) and (b) indicate only the harmonics generated by the upconversion step, i.e., assuming an unmodulated laser output of $E_{1}(t)=\sqrt{2 P_{i n}} e^{j \omega_{c} t}$ in Equations (20) and (21), where $f_{c}=\frac{\omega_{c}}{2 \pi}$ and $P_{i n}$ are the laser frequency and optical power, respectively. Any data employed in the prior baseband/IF modulation step would be located around the harmonics. b) Maximum Transmission Point (MATP) Biasing: This technique requires the MZM of Fig. 26(a) to be biased at a voltage of $V_{\text {bias }}=\left(0+m 2 V_{\pi}\right)$, where $m$ is an integer. As seen from Fig. 26(b), this bias point corresponds to the maximum output optical power and hence it is referred to as the Maximum Transmission Point (MATP) [68]. Using Equations (2b) and (2c), the expressions for the optical field $E_{2}(t)$ at the output of the biased MZM in Fig. 26(a) and the photo-detected signal $I(t)$ in the MATP biasing case can be represented as follows:

$$
\begin{aligned}
& E_{2}(t)=\cos \left(\frac{\pi V_{L O} \cos \left(\omega_{L O} t\right)}{2 V_{\pi}}\right) E_{1}(t) \\
& =\frac{E_{1}(t)}{\sqrt{2}}\left[J_{0}\left(\frac{\pi V_{L O}}{2 V_{\pi}}\right)+2 \sum_{n=1}^{\infty}(-1)^{n} J_{2 n}\left(\frac{\pi V_{L O}}{2 V_{\pi}}\right) \cos \left(2 n \omega_{L O} t\right)\right]
\end{aligned}
$$

and

$$
\begin{aligned}
I(t) \propto\left|E_{2}(t)\right|^{2}= & \frac{\left|E_{1}(t)\right|^{2}}{2}\left[1+\cos \left(\frac{\pi V_{L O} \cos \left(\omega_{L O} t\right)}{V_{\pi}}\right)\right] \\
= & \frac{\left|E_{1}(t)\right|^{2}}{2}\left[1+J_{0}\left(\frac{\pi V_{L O}}{V_{\pi}}\right)\right. \\
& \left.+2 \sum_{n=1}^{\infty}(-1)^{n} J_{2 n}\left(\frac{\pi V_{L O}}{V_{\pi}}\right) \cos \left(2 n \omega_{L O} t\right)\right] .
\end{aligned}
$$

Observe from Equation (22) that the optical field has only even harmonics, i.e., optical harmonics of the order $2 n$, where $n=0,2, \ldots$, as shown in Fig. 27(c). Additionally, it can be seen from Equation (23) that the photo-detected signal consists of even harmonics values, i.e., $N=2 k$, where $k=0,1,2, \ldots$, as shown in Fig. 27(d). Note that Fig. 27(c) and (d) indicate only the harmonics generated by the upconversion step, i.e., assuming an unmodulated laser output of $E_{1}(t)=\sqrt{2 P_{i n}} e^{j \omega_{c} t}$ in Equations (22) and (23), while any data employed in the prior baseband/IF modulation step would be located around the harmonics.

c) Minimum Transmission Point (MITP) Biasing: This technique requires the MZM in Fig. 26(a) to be biased at a voltage of $V_{\text {bias }}=V_{\pi}+m 2 V_{\pi}$, where $m$ is an integer. As seen from Fig. 26(b), this bias point corresponds to the minimum output optical power and hence it is referred to as the Minimum Transmission Point (MITP) [68]. Using Equations (2b) and (2c), the expressions for the optical 
field $E_{2}(t)$ at the output of the biased MZM in Fig. 26(a) and the photo-detected signal $I(t)$ in the MITP biasing case are as follows:

$$
\begin{aligned}
& E_{2}(t) \\
& =\mp \sin \left(\frac{\pi V_{L O} \cos \left(\omega_{L O} t\right)}{2 V_{\pi}}\right) E_{1}(t) \\
& = \pm \frac{E_{1}(t)}{\sqrt{2}}\left[2 \sum_{n=1}^{\infty}(-1)^{n} J_{2 n-1}\left(\frac{\pi V_{L O}}{2 V_{\pi}}\right) \cos \left((2 n-1) \omega_{L O} t\right)\right]
\end{aligned}
$$

and

$$
\begin{aligned}
I(t) & \propto\left|E_{2}(t)\right|^{2} \\
= & \frac{\left|E_{1}(t)\right|^{2}}{2}\left[1-\cos \left(\frac{\pi V_{L O} \cos \left(\omega_{L O} t\right)}{V_{\pi}}\right)\right] \\
= & \frac{\left|E_{1}(t)\right|^{2}}{2}\left[1-J_{0}\left(\frac{\pi V_{L O}}{V_{\pi}}\right)\right. \\
& \left.-2 \sum_{n=1}^{\infty}(-1)^{n} J_{2 n}\left(\frac{\pi V_{L O}}{V_{\pi}}\right) \cos \left(2 n \omega_{L O} t\right)\right] .
\end{aligned}
$$

Observe from Equation (24) that the optical field has only odd harmonics, i.e., optical harmonics of the order $(2 n-$ 1 ), where $n=1,2, \ldots$, as shown in Fig. 27(e). Additionally, it can be seen from Equation (25) that the photodetected signal consists of even harmonics values, i.e., $N=2 n$ where $n=0,1,2, \ldots$, as shown in Fig. 27(f). Note that Fig. 27(e) and (f) indicate only the harmonics generated by the upconversion step, i.e., assuming an unmodulated laser output of $E_{1}(t)=\sqrt{2 P_{i n}} e^{j \omega_{c} t}$ in Equation (24) and Equation (25), while any data employed in the prior baseband/RF modulation step would be located around the harmonics.

This technique is also referred to as Optical Carrier Suppression (OCS), because the optical carrier at $\omega_{c}$ is suppressed in the modulated signal, as shown in Fig. 27(e) [30]. The modulation voltage $V_{L O}$ is much smaller than the switching voltage $V_{\pi}$ of the MZM, hence in Equation (25), we have $\mathrm{J}_{0}\left(\frac{\pi V_{L O}}{V_{\pi}}\right) \approx 1$. Therefore, unlike in the MATP and QP biasing schemes of this section, the DC component in the photo-detected signal of MITP biasing, i.e., the term $\left[1-J_{0}\left(\frac{\pi V_{L O}}{V_{\pi}}\right)\right]$ in Equation (25), is highly suppressed. Hence, upon using Equations (10) and (11), it can be concluded that an advantage of a suppressed DC component is a low RIN and shot noise [71]. Furthermore, compared to the other N-tupling biasing techniques and the previously discussed OSSB and ODSB modulation, OCS has a better receiver sensitivity [65], [72].

Several values of $N$ have been employed in the literature for $\mathrm{N}$-tupling such as in doubling with $N=2$, tripling with $N=3$, quadrupling with $N=4$, quintupling with $N=5$, six-tupling with $N=6$ and 12-tupling with $N=12$. These implementations rely on the previously discussed biasing techniques. In

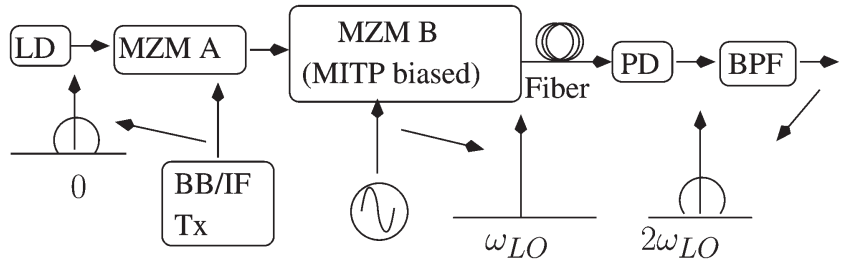

Fig. 28. Frequency doubling using Minimum Transmission Point (MITP) biasing (or Optical Carrier Suppression (OCS)).

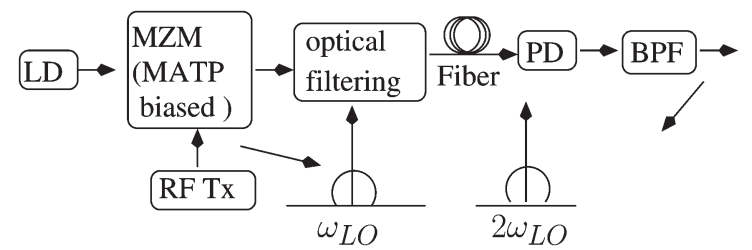

Fig. 29. Frequency doubling using Maximum Transmission Point (MATP) biasing.

the following discussion, we present some of the commonly employed optical $\mathrm{N}$-tupling implementations.

a) Frequency doubling: Frequency doubling refers to the scenario where the photo-detected signal has a carrier frequency that is twice that of the transmitted signal. Frequency doubling can be achieved in the following ways:

- Fig. 28 shows the block diagram of frequency doubling using MITP biasing, where the output of a laser in the BS is modulated using the $f_{I F}=\frac{\omega_{I F}}{2 \pi}$ $\mathrm{Hz}$ baseband/IF electronic signal by the first MZM, namely MZM A [30]. When the second MZM of Fig. 28, namely MZM B, is biased at the MITP and driven by an electronic tone at $f_{L O}=\frac{\omega_{L O}}{2 \pi} \mathrm{Hz}$, the optical field consists of a suppressed carrier and odd harmonics, where the strongest ones are the two first-order harmonics on either side of the carrier, as shown in Fig. 27(e). During photo-detection in the RAP, these first order harmonics beat together to generate a signal, whose center frequency is $f_{R F}=$ $\left(2 f_{L O}+f_{I F}\right) \mathrm{Hz}$, i.e., the center frequency is twice that of the modulating electronic tone [30]. This technique has been employed both in generating $40 \mathrm{GHz}$ [30] and $60 \mathrm{GHz}$ signals [52].

- Fig. 29 shows the block diagram of frequency doubling using MATP biasing [73], where frequency doubling is carried out by feeding the output of a laser operating in the $\mathrm{BS}$ at $f_{c}=\frac{\omega_{c}}{2 \pi} \mathrm{Hz}$ to a MATP biased MZM that is driven by a RF signal at $f_{L O}=\frac{\omega_{L O}}{2 \pi} \mathrm{Hz}$, in order to generate the spectrum of Fig. 27(c). Afterwards, the second-order optical harmonic at $\left(f_{c}-2 f_{L O}\right) \mathrm{Hz}$ will be eliminated by the optical filter in Fig. 29 to generate a dispersion tolerant OSSB signal having a single second order harmonic at $\left(f_{c}+2 f_{L O}\right) \mathrm{Hz}$. Subsequently, photodetection in the RAP generates a frequency-doubled electronic signal, because the single sideband at $\left(f_{c}+2 f_{L O}\right) \mathrm{Hz}$ beats with the optical carrier at $f_{c} \mathrm{~Hz}$. 


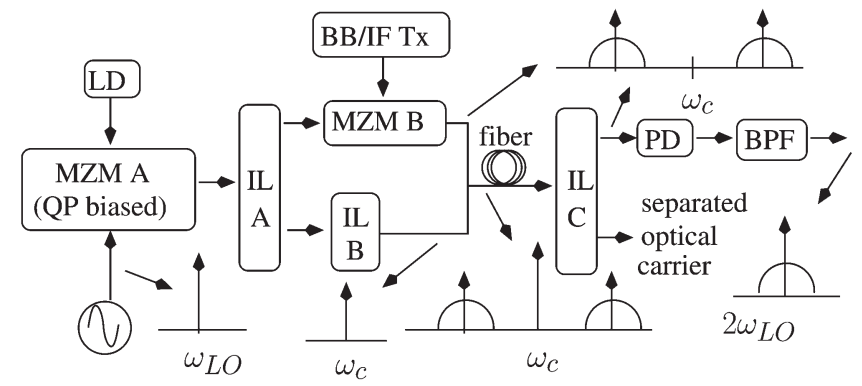

Fig. 30. Frequency doubling using quadrature biasing (IL-Interleaver).

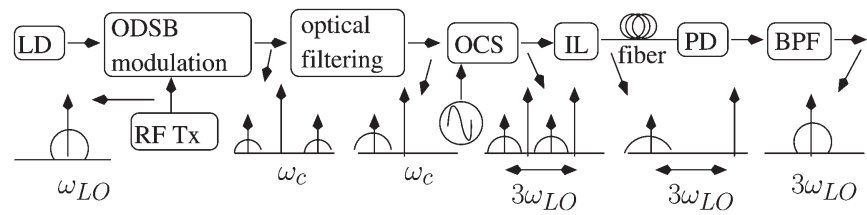

Fig. 31. Frequency tripling.

- Fig. 30 shows the block diagram of frequency doubling using QP biasing, where frequency doubling can be achieved by feeding the output of a laser operating in the $\mathrm{BS}$ at $f_{c}=\frac{\omega_{c}}{2 \pi} \mathrm{Hz}$ to a quadrature point biased MZM, namely MZM A that is driven by a RF tone at $f_{L O}=\frac{\omega_{L O}}{2 \pi} \mathrm{Hz}$ [74], [67]. The output of MZM A, whose spectrum is shown in Fig. 27(a), is fed to the interleaver IL A of Fig. 30, which separates the odd optical harmonics from the even ones, where the most significant odd optical harmonics are the two first order harmonics at $\left(\omega_{c} \pm \omega_{L O}\right)$ [74], which are modulated by the baseband/IF data using the second MZM, namely MZM B. The even optical harmonics primarily consist of the optical carrier and the second order harmonics. The pure optical carrier is separated using IL B. The optical carrier and the modulated first-order harmonics are then multiplexed together and transmitted over the fiber to the RAP. At the RAP, the optical carrier is separated out using IL C of Fig. 30, while the two first-order harmonics beat together during photo-detection in order to generate a frequency-doubled electronic signal [74]. The separated carrier of Fig. 30 can then be re-used for achieving uplink communication, which is known as wavelength-reuse.

b) Frequency tripling: Frequency tripling can be achieved by employing two MZMs in the upconversion step, as shown in the BS of Fig. 31 [75], where the laser output at $f_{c}=\frac{\omega_{c}}{2 \pi} \mathrm{Hz}$ enters the first modulator, which is a MZMbased ODSB modulator that is driven by a RF signal at $f_{L O}=\frac{\omega_{L O}}{2 \pi} \mathrm{Hz}$. The optical output of the ODSB modulator seen in Fig. 31 consists of the optical carrier and strong first-order harmonics. The first optical modulation is then followed by elimination of one of the first-order harmonics by the optical filter of Fig. 31. Afterwards, the filtered signal enters the second MZM, which implements OCS, i.e., it is biased at the MITP [75], and is driven by a $\mathrm{RF}$ tone at $f_{L O} \mathrm{~Hz}$, thereby generating a signal

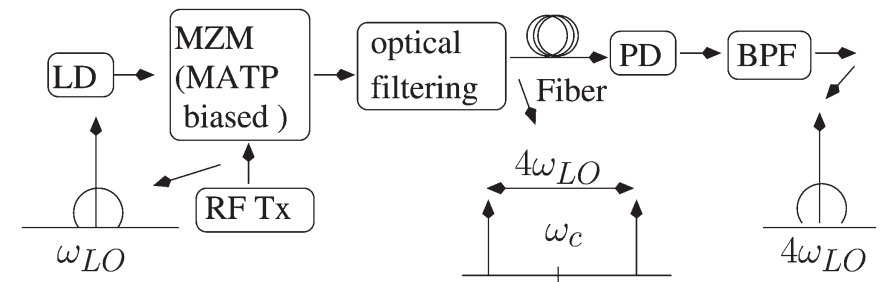

Fig. 32. Frequency quadrupling by Maximum Transmission Point (MATP) biasing.

whose optical spectrum is shown in Fig. 31. As portrayed in Fig. 31, the interleaver (IL) selects the $3 \omega_{L O}$ spaced harmonics, which are then transmitted over the fiber to the RAP. At the RAP, the $3 \omega_{L O} \mathrm{~Hz}$ separated harmonics beat together during photo-detection to generate a frequencytripled signal at $3 f_{L O} \mathrm{~Hz}$.

c) Frequency quadrupling: Frequency quadrupling can be implemented either using a MATP biased MZM or by using a nested-MZM, as discussed below:

- Fig. 32 shows the block diagram of frequency quadrupling using MATP biasing, where the output of the laser operating in the BS at $f_{c}=\frac{\omega_{c}}{2 \pi} \mathrm{Hz}$ is fed to an MATP-biased MZM whose output spectrum is shown in Fig. 27(c). Then, the optical carrier frequency in Fig. 27(c) is eliminated using a notch filter and then the remaining signal is transmitted over the fiber to the RAP. The subsequent photo-detection of the two $4 \omega_{L O}$-spaced second-order harmonics seen at $\omega_{c} \pm 2 \omega_{L O}$ in Fig. 27(c) produces a frequencyquadrupled signal, as shown in Fig. 32 [70]. This method has been employed for generating signals that are tunable from 32 to $50 \mathrm{GHz}$ [70]. Instead of employing a notch filter at the BS, an interleaver may be employed at the RAP in a manner similar to that in Fig. 30 [76]. One of the outputs of the interleaver would consist of the second order harmonics, while the other output would be the separated carrier. The second-order harmonics are photo-detected for generating a frequency-quadrupled signal, while the separated carrier may be employed by the abovementioned wavelength re-use technique [73], [76], where again, wavelength re-use refers to the employment of the received downlink optical signal for uplink optical transmission.

- An alternative to applying an optical filter in Fig. 32 is to carefully set the depth of the optical modulation using the electronic $\mathrm{LO}$, which is also referred to as the LO modulation index, to a value that suppresses the optical carrier [77], i.e., to ensure that $J_{0}\left(\frac{\pi V_{L O}}{2 V_{T}}\right)=0$ in Equation (22), which yields $V_{L O}=\left(2 V_{\pi} / \pi\right) * 2.405$ [77]. However, at this LO modulation index, the strength of the optical harmonics in Equation (22), that are above the secondorder harmonics, become significant [77].

- Carrier suppression can also be achieved by feeding the output of a laser operating in the BS at $f_{c}=\frac{\omega_{c}}{2 \pi} \mathrm{Hz}$ to a nested MZM [67], [78], as shown 


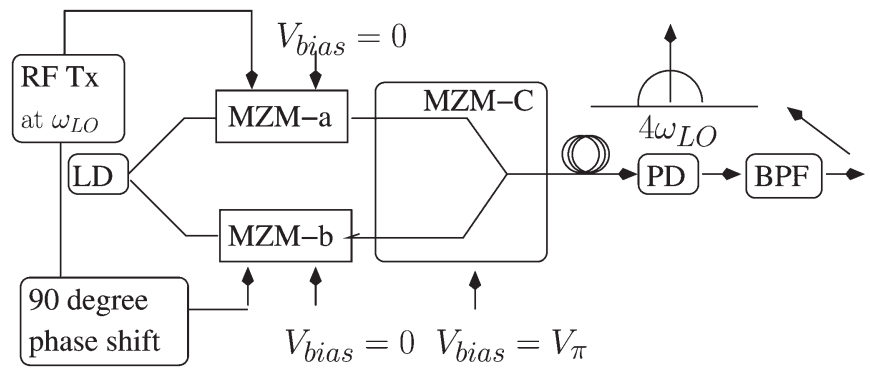

Fig. 33. Frequency quadrupling by nested Mach Zehnder Modulator (MZM).

in Fig. 33. In Fig. 33, the first two MZMs, namely MZM-a and MZM-b, are biased at $V_{\text {bias }}=0$ and driven by the signals $V_{\text {drive, } a}=V_{R F} \sin \omega_{L O} t$ and $V_{\text {drive }, b}=V_{R F} \cos \omega_{L O} t$, respectively. Hence, using Equation (2b), the outputs of MZM-a and MZM-b are [78]:

$$
\begin{aligned}
E_{a}(t)= & \cos \left(\frac{\pi V_{L O} \sin \omega_{L O} t}{V_{\pi}}\right) \sqrt{2 P_{i n}} e^{j \omega_{c} t} \\
= & {\left[J_{0}\left(\frac{\pi V_{L O}}{V_{\pi}}\right)\right.} \\
& \left.+2 \sum_{k=1}^{\infty} J_{2 k}\left(\frac{\pi V_{L O}}{V_{\pi}}\right) \cos \left(2 k \omega_{L O} t\right)\right] \sqrt{2 P_{i n}} e^{j \omega_{c} t}
\end{aligned}
$$

and

$$
\begin{aligned}
E_{b}(t)= & \cos \left(\frac{\pi V_{L O} \cos \omega_{L O} t}{V_{\pi}}\right) \sqrt{2 P_{i n}} e^{j \omega_{c} t} \\
= & {\left[J_{0}\left(\frac{\pi V_{L O}}{V_{\pi}}\right)\right.} \\
& \left.+2 \sum_{k=1}^{\infty}(-1)^{k} J_{2 k}\left(\frac{\pi V_{L O}}{V_{\pi}}\right) \cos \left(2 k \omega_{L O} t\right)\right] \sqrt{2 P_{i n}} e^{j \omega_{c} t} .
\end{aligned}
$$

As shown in Fig. 33, the outputs of MZM-a and MZM-b are fed to the third MZM, namely MZM-c, which is biased at $V_{\text {bias }}=V_{\pi}$. Hence, resulting in:

$$
\begin{aligned}
E_{c}(t) & =\frac{E_{a}(t)+e^{j \frac{\pi V_{b i a s}}{V_{\pi}}} E_{b}(t)}{2}=\frac{E_{a}(t)-E_{b}(t)}{2} \\
& =\frac{1}{2} \sum_{k=1}^{\infty} J_{4 k-2}\left(\frac{\pi V_{R F}}{V_{\pi}}\right) \cos \left((4 k-2) \omega_{L O} t\right) \sqrt{2 P_{\text {in }}} e^{j \omega_{c} t}
\end{aligned}
$$

Thus, $E_{c}(t)$ has harmonics at $\omega_{c} \pm(4 k-2) \omega_{L O}$, of which the ones at $\left(\omega_{c} \pm 2 \omega_{L O}\right)$ are the strongest. These harmonics beat during photo-detection in the RAP for generating a frequency-quadrupled signal at $4 \omega_{L O}$.

d) Frequency quintupling: Fig. 34 shows the block diagram of frequency quintupling, which can be achieved by employing two stages. The output of a laser operating in the BS at $f_{c}=\frac{\omega_{c}}{2 \pi} \mathrm{Hz}$ enters the first modulation stage,

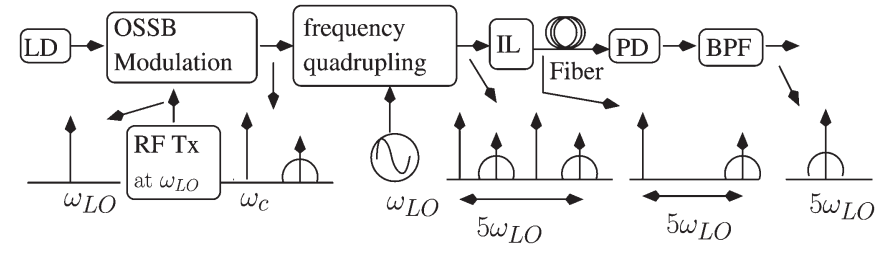

Fig. 34. Frequency quintupling.

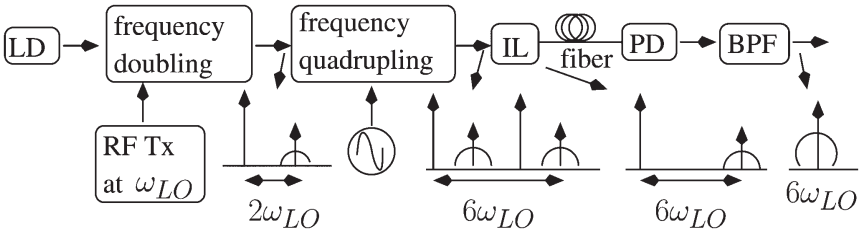

Fig. 35. Frequency six-tupling.

which is driven by a RF signal at $f_{L O}=\frac{\omega_{L O}}{2 \pi} \mathrm{Hz}$ and it generates an OSSB signal, as shown in Fig. 34. The second stage is driven by a $\mathrm{RF}$ tone at $f_{L O} \mathrm{~Hz}$ and it implements frequency quadrupling, which generates an optical signal whose spectrum is shown in Fig. 34. The interleaver of Fig. 34 then separates the $5 \omega_{L O}$-spaced sidebands, and these sidebands beat during the photodetection in the RAP to generate a frequency-quintupled signal [67].

e) Frequency six-tupling: Frequency six-tupling refers to the technique where the PD's output signal has a carrier frequency that is six times that of the transmitted RF signal. Diverse architectures may be used for frequency six-tupling.

First, the previously discussed OFM architecture of Fig. 24(c) can be employed for achieving frequency six-tupling. The discriminator commonly used in OFM assisted N-tupling is an MZI [79]. The photo-detected signal of Fig. 24(c) includes the upconverted signals corresponding to all values of $N$, where frequency sixtupling can be achieved by filtering out the sideband at $N=6$, resulting in $\omega_{R F}=6 \omega_{L O}$. Additionally, Fig. 35 shows another block diagram designed for achieving frequency six-tupling in two stages. The output of the laser in the BS is fed to the first stage, which implements frequency doubling by generating a frequency-doubled OSSB signal. The output of the first stage is then fed to the second stage, which implements frequency quadrupling, thereby generating an optical signal whose spectrum is shown in Fig. 35. The $6 \omega_{L O}$-spaced sidebands are separated using an interleaver and are transmitted to the RAP over the optical fiber. At the RAP, these sidebands beat during photo-detection for generating a frequency sixtupled signal [67].

f) Frequency 12-tuping: Fig. 36 shows a block diagram conceived for frequency 12-tupling, which can be achieved by feeding the output of a laser operating in the BS at $f_{c}=\frac{\omega_{c}}{2 \pi} \mathrm{Hz}$ to a frequency quadrupling stage. This generates $4 \omega_{L O}$-spaced sidebands at $\omega_{C} \pm 2 \omega_{L O}$, as shown in Fig. 36. Subsequently, the FWM in a Semiconductor Optical Amplifier (SOA) results in the generation 


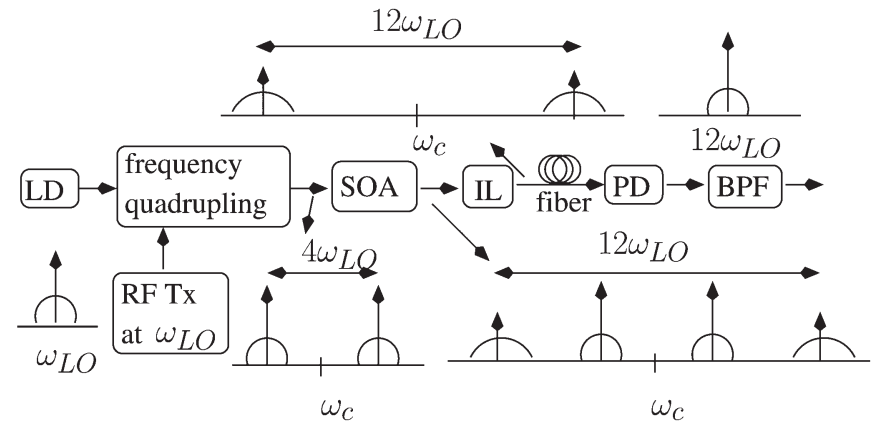

Fig. 36. Frequency 12-tupling (SOA-Semiconductor Optical Amplifier).

of new optical frequencies at $\left(\omega_{c} \pm 6 \omega_{L O}\right)$ according to Equation (8). These $12 \omega_{L O}$-spaced sidebands are then separated using the interleaver of Fig. 36. Then, during photo-detection in the RAP, the beating of these $12 \omega_{L O}$-spaced sidebands generates a frequency 12 -tupled signal [80].

The discussion presented so far in this section assumed having an ideal extinction ratio of $\gamma=1$ in Equation (3), because a non-ideal extinction-ratio results in imperfect attenuation of the suppressed harmonics in the optical field and hence reduces the power of the desired harmonics. For example, Equation (24) states that the optical field has only odd harmonics for case of MITP biasing. However, when we have $\gamma \approx 1$, the optical field also has imperfectly suppressed even harmonics.

2) Design Guidelines When Using Optical N-Tupling Techniques: The photo-detected signal power at a frequency of $f_{R F}$ suffers from a Dispersion Induced Power Penalty (DIPP) due to the fiber's chromatic dispersion [68], where the extent of the power penalty depends on the modulation index of the modulation by the LO [68]. The distortion is minimized for the optimum values of the LO oscillator modulation index, for which the expression for DIPP can be simplified by neglecting non-dominant terms as follows [68]:

1) For QP biasing:

$$
D I P P \approx \cos \left(\beta f_{I F} f_{R F}\right) \cos \left(\beta f_{L O} f_{R F}\right) ;
$$

2) For MATP biasing

$$
\begin{aligned}
D I P P & \approx \cos \left(\beta f_{I F} f_{R F}\right) \quad \forall N=4+4 k \\
& \approx \cos \left(\beta f_{I F} f_{R F}\right) \cos \left(2 \beta f_{L O} f_{R F}\right) \quad \forall N=2+4 k ;
\end{aligned}
$$

\section{3) For MITP biasing}

$$
\begin{aligned}
D I P P & \approx \cos \left(\beta f_{I F} f_{R F}\right) \quad \forall N=2+4 k \\
& \approx \cos \left(\beta f_{I F} f_{R F}\right) \cos \left(2 \beta f_{L O} f_{R F}\right) \quad \forall N=4+4 k ;
\end{aligned}
$$

where we have $\beta=\left(\pi D \lambda_{c}^{2} L\right) / c, D$ is the chromatic dispersion parameter, $L$ is the length of the optical fiber, $c$ is the speed of light in vacuum and $\lambda_{c}$ is the optical carrier wavelength.
It is inferred from Equation (29) that when biasing at the MATP, it is better to employ photo-detected harmonics of the order $N=(4+4 K)$ in order to minimize the DIPP. On the other hand, observe from Equation (30) that it is better to employ photo-detected harmonics of the order $N=(2+4 K)$ [68], when biasing at MITP, in order to minimize the DIPP. In general, the extent of DIPP, when biasing is done at MATP or MITP is less than that for the case of QP biasing [81]. The values of $f_{I F}$ and $f_{R F}$ are decided by the specifications of the network for which the ROF link is the backhaul. Thus, observe from Equation (19) that there is an $N$ versus $f_{L O}$ trade-off, where a higher $N$ results in the ability to employ a lower $f_{L O}$ [68] and hence reducing the electrical bandwidth requirement of the MZM. On the other hand, when $N$ increases, the strength of the LO signal that is required for generating a strong harmonic increases and the achievable strength of the upconverted photo-detected signal also decreases. Thus, for large values of $N$, a MZM that can handle high values of drive power is required. For example, a peak-to-peak amplitude larger than twice the switching voltage may be needed in the case of frequency quadrupling for achieving the desired performance [82]. One of the solutions proposed in [82] was to achieve frequency quadrupling by performing frequency doubling twice [82]-[84].

The N-tupling techniques employ optical harmonics instead of optical inter-modulation, where the difference between the two was detailed in Section III-C1. As discussed in Section III-C1, the undesired effect of optical inter-modulation occurs when a multi-channel, i.e., multi-frequency, modulating signal is employed in optical modulation. Upon comparing the transmittance curves of Figs. 5(b) and 8, it can be stated that the more non-linear transfer function of the MZM results in the generation of stronger inter-modulation products, as compared to direct modulation. Single-step N-tupling in a multichannel scenario involves the MZM assisted modulation using multiplexed RF signals and hence has strong optical intermodulation products [58]. By contrast, the inter-modulation signals are weaker in 2-step N-tupling, because multiplexed IF signals can be employed in the more linear direct modulation during the first step, while the subsequent MZM assisted upconversion step uses a single-frequency LO tone. Additionally, one of the major advantages of such a two-step implementation of optical N-tupling can be observed in a WDM system, as shown in Fig. 37, which requires photo-detected signals to be generated at high RF carriers [85]. Direct modulation of the laser diodes cannot be readily achieved using RF signals at high frequencies, while the employment of an external modulator for each source is expensive. Hence, as shown in Fig. 37, the lasers are directly modulated with their respective baseband/IF data and then the multiplexed outputs are fed into a MZM modulator, that is driven by a local oscillator at $f_{L O} \mathrm{~Hz}$ for simultaneous upconversion [65]. This ensures that each wavelength carries an upconverted optical signal. However, 2-step $\mathrm{N}$-tupling results in an increased transmitter cost, since there are two modulation steps.

Furthermore, the two-step N-tupling may also be performed in the reverse order as shown in Fig. 30, where modulation using a LO (Upconversion step) followed by the modulation 


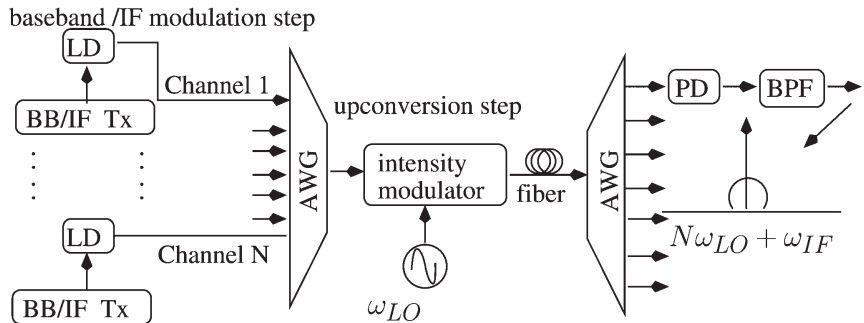

Fig. 37. Optical upconversion of Wavelength Division Multiplexing (WDM) signal (AWG-Arrayed Waveguide Grating).

using the IF/baseband data is employed [74]. In this case the first modulation is RF and it may be beyond the direct modulation bandwidth of the laser to be used. Hence, such an ordering may require both steps to be based on external modulation.

According to the discussions in Section IV-A1, the photodetected signal consists of either only odd or only even harmonics, when intensity modulation based N-tupling is employed. However, the OFM technique illustrated in Fig. 24(c) can employ any value of $N$ [69], which provides a greater flexibility for the designer, including the ability to implement dynamic radio channel assignment [86], [69]. Explicitly, the designer can dynamically generate the wireless signal at any frequency, which is a multiple of $f_{L O} \mathrm{~Hz}$.

Having discussed the subclass of optical N-tupling techniques within the family of techniques in Fig. 22 that rely on the non-linearity of the MZM for optical upconversion, we now move on to discussing the next subclass within the same family of techniques. Optical N-tupling relied on optical harmonics for achieving optical upconversion. By contrast, the next subclass of techniques achieves optical upconversion by relying on optical inter-modulation, as shown in Fig. 22.

3) Optical Upconversion Relying on Optical InterModulation: It can be inferred from the transmittance curve of Fig. 8 that the highly non-linear transfer function of the MZM results in the generation of strong inter-modulation products, which can be exploited for achieving optical upconversion. The baseband/IF signal at $f_{I F}=\frac{\omega_{I F}}{2 \pi} \mathrm{Hz}$ is added to the local oscillator signal at $f_{L O}=\frac{\omega_{L O}}{2 \pi} \mathrm{Hz}$, where the combined drive signal of $V(t)=V_{I F} \cos \left(\omega_{I F} t\right)+V_{L O} \cos \left(\omega_{L O} t\right)$ is invoked for driving the MZM in the BS that is biased at MITP for achieving OCS intensity modulation, i.e., we have $V_{\text {bias }}=$ $V_{\pi}+m 2 V_{\pi}$, where $m$ is an integer. Using Equation (2c) and the Taylor series expansion of $\cos (x)$ [87], the intensity of the output is given by:

$$
\begin{aligned}
P(t)= & P_{i n}\left[1-\cos \left(\frac{\pi V(t)}{V_{\pi}}\right)\right], \\
= & P_{i n}\left[1-\left(1-\frac{1}{2}\left(\frac{\pi V(t)}{V_{\pi}}\right)^{2}+\text { weaker terms }\right)\right] \\
\approx & P_{i n} \frac{\pi^{2}}{2 V_{\pi}^{2}}\left[V_{I F}^{2} \cos ^{2} \omega_{I F} t\right. \\
& \left.+V_{L O}^{2} \cos ^{2} \omega_{L O} t+2 V_{I F} V_{L O} \cos \omega_{I F} t \cos \omega_{L O} t\right]
\end{aligned}
$$

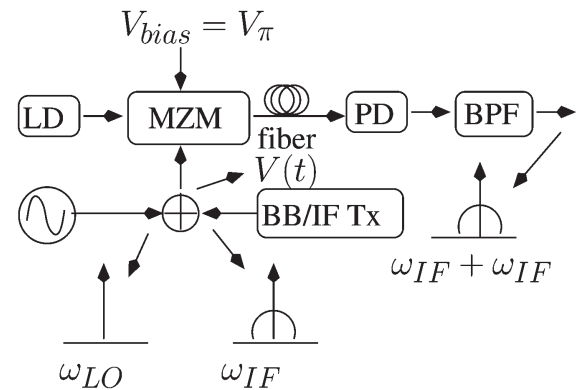

Fig. 38. Optical upconversion using optical inter-modulation.

The third term in Equation (31) generates modulated RF signals at $\omega_{R F}=\left(\omega_{L O} \pm \omega_{I F}\right)$. As mentioned previously, the photo-detected signal is proportional to the optical intensity. Hence, in the RAP, the upconverted signals at $\omega_{R F}=\left(\omega_{L O} \pm\right.$ $\omega_{I F}$ ) can be retained from the photo-detected signal by the BPF of Fig. 38 [88], [89]. Biasing at MATP also generates photodetected signals at $\omega_{R F}=\left(\omega_{L O} \pm \omega_{I F}\right)$, but the achievable optical SNR is higher if biasing is done at MITP than for MATP owing to the former's reduced RIN noise [71]. Hence the MZM performs the function of a mixer along with that of an optical modulator. This enables the ROF system to avoid the use of high RF mixers, which would have otherwise degraded the BER of wireless communication.

The optical upconversion techniques discussed so far exploited the non-linearity of the MZM situated at the transmitter. Let us now discuss the family of wavelength-conversion based optical upconversion techniques shown in Fig. 22, which rely on the non-linearity of other optical network components, such as the optical fiber, the Semiconductor Optical Amplifier (SOA) and the Electro-Absorption Modulator (EAM).

\section{B. Wavelength-Conversion Based Optical Upconversion}

Modulating the laser's $f_{c}=\frac{\omega_{c}}{2 \pi} \mathrm{Hz}$ output using a MZM biased at the MITP and driven by a RF tone at $f_{L O} / 2 \mathrm{~Hz}$ generates an optical signal that consists of odd harmonic frequencies with the first harmonics at $\omega_{c} \pm\left(\omega_{L O} / 2\right)$ being dominant. This signal has the spectrum shown in Fig. 40 and is referred to as a $f_{L O} \mathrm{~Hz}$ LO Optical Signal (LOOS) because its photo-detection generates a LO electronic signal at the difference frequency of $f_{L O}=\frac{\omega_{L O}}{2 \pi} \mathrm{Hz}$. It must be noted that a $f_{L O} \mathrm{~Hz}$ LOOS at $f_{L O O S} \mathrm{~Hz}$ is located at an optical frequency of $f_{L O O S} \mathrm{~Hz}$ and generates a $f_{L O} \mathrm{~Hz}$ electronic tone on photo-detection.

Optical wavelength conversion refers to the process of copying the transmitted data from one wavelength at $f_{c}=\frac{\omega_{c}}{2 \pi} \mathrm{Hz}$ to another at $f_{L O O S}=\frac{\omega_{L O O S}}{2 \pi} \mathrm{Hz}$. If the wavelength at $\omega_{C}$ carries the baseband/IF data signal, while the wavelength at $\omega_{\text {LOOS }}$ carries a LOOS prior to wavelength conversion, then the process of wavelength conversion also results in optical upconversion, i.e., the photo-detection of the output optical signal generates the upconverted data signal at $f_{L O} \mathrm{~Hz}$.

Wavelength conversion based non-linear techniques facilitate cost savings with the aid of wavelength re-use [90] and does not rely on the polarization-sensitive MZM. Additionally, the ability to move data from one wavelength to another facilitates optical switching [37], while using a $f_{\text {LOOS }} \mathrm{Hz}$ wavelength 


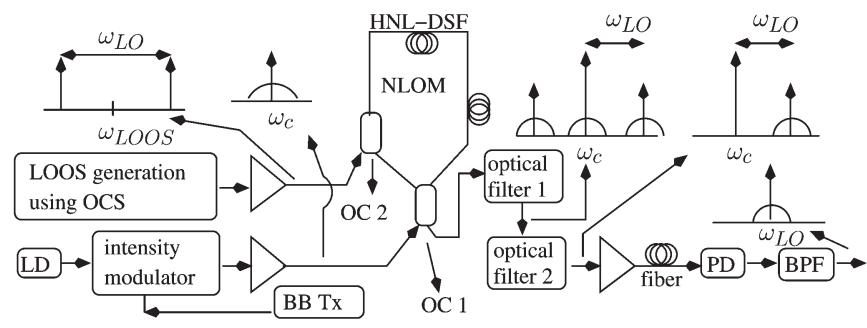

Fig. 39. Cross Phase Modulation (XPM) assisted upconversion (HNLDSF_-Highly Nonlinear Dispersion-Shifted Fiber).

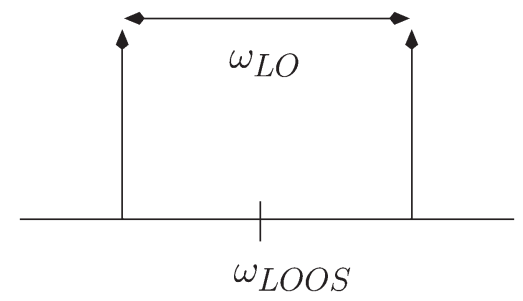

Fig. 40. Local Oscillator Optical Signal (LOOS).

having different values of the $f_{L O} \mathrm{~Hz}$ LOOS enables wireless frequency selection in WDM-ROF networks. Wavelength conversion techniques exploit the non-linearity of fibers [61], of the SOA [63] or EAM [64], as discussed in the following sections.

1) Techniques Based on the Non-Linearity of the Fiber: As discussed in Section II-B3, the dependence of the fiber's refractive index on the optical power introduces a non-linear phenomenon referred to as XPM. Additionally, it was also mentioned in Section II-B3 that a second strong non-linear phenomenon known as FWM is present, when the dispersion parameter $D$ is small. Most ROF optical systems operate at $1550 \mathrm{~nm}$ because of the low fiber attenuation at that wavelength, while zero dispersion occurs at $1300 \mathrm{~nm}$ [37]. A Highly Nonlinear Dispersion-Shifted Fiber (HNL-DSF) is a special fiber that has a highly non-linear refractive-index, while the zerodispersion wavelength is shifted from $1300 \mathrm{~nm}$ to $1550 \mathrm{~nm}$. In the context of wavelength conversion techniques, the signals are propagated through a certain length of the HNL-DSF in order to exploit these non-linear phenomena.

Fig. 39 shows an XPM-assisted optical upconversion architecture, where, in the BS, the laser output at $\omega_{c}$ is modulated with the baseband data and then this signal is injected into a Non-linear Optical Loop Mirror (NOLM) ${ }^{8}$ at OC 1 [61]. As shown in Fig. 39, a LOOS at $\omega_{\text {LOOS }}$ is injected into the loop at OC 2, where the LOOS modulates the phase of the two counterpropagating $\omega_{c}$-frequency signals through XPM [61]. The two counter-propagating, phase modulated $\omega_{c}$-frequency waves are then combined again at OC 1 after propagating through the entire loop. The intensity of the combined signal depends on the phase difference of the two waves. Thus, the NOLM induces XPM in the signal at $\omega_{c}$ using the signal at $\omega_{\text {LOOS }}$ and then performs phase-to-intensity conversion. Hence, the NOLM output consists of the LOOS at $\omega_{\text {LOOS }}$ and the XPM-

\footnotetext{
${ }^{8} \mathrm{~A}$ NOLM is an architecture where the input signal at $\omega_{c}$ is split into two waves by the coupler OC 1, as shown in Fig. 39. These two waves propagate in opposite directions (clockwise and counter-clockwise) in a fiber-loop made from HNL-DSF and hence acquire different phases due to fiber non-linearity.
}

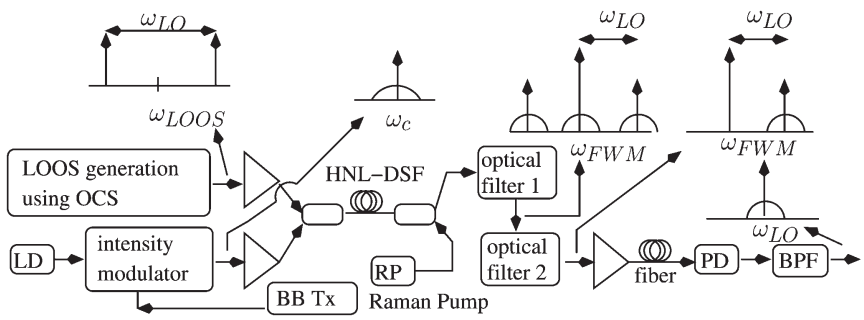

Fig. 41. Four Wave Mixing (FWM) assisted upconversion (RP-Raman Pump).

assisted intensity-modulated signal at $\omega_{c}$. The spectrum of the intensity modulated output of the NLOM at $\omega_{c}$ is similar to an ODSB signal, where Fig. 39 shows the ODSB spectrum after the elimination of the LOOS at $\omega_{\text {LOOS }}$ using optical filter 1 [61]. Afterwards, one of the sidebands of the ODSB signal is eliminated by optical filter 2 for generating an OSSB signal. The optical frequencies of $f_{L O O S}=\frac{\omega_{L O O S}}{2 \pi} \mathrm{Hz}$ and $f_{c}=\frac{\omega_{C}}{2 \pi}$ $\mathrm{Hz}$ are not sufficiently close to each other for enabling the use of a single filter, instead of the two step filtering. The OSSB signal is then amplified, transmitted over the ROF link to the RAP and photo-detected for generating a RF signal at $\omega_{L O}$. Although the architecture of Fig. 39 employs a single basebanddata modulated wavelength at $\omega_{c}$, this concept can be extended to a WDM signal that is generated by combining the datamodulated outputs of several lasers. This WDM signal would then be combined with the LOOS at $\omega_{\text {LOOS }}$ in a NLOM and employed for optical upconversion [61].

Additionally, the FWM can be employed for achieving optical upconversion [91]. Fig. 41 shows the block diagram of an architecture that implements optical upconversion using FWM, where, in the BS, the laser output at $\omega_{c}$ is modulated by the baseband data. The modulated signal is then injected into the HNL-DSF of Fig. 41 along with a LOOS at $\omega_{\text {LOOS }}$, as shown in Fig. 41. The FWM in the fiber generates new signals as per Equations (7) and (8), where the spectrum of the signal at the output of the HNL-DSF is shown in Fig. 42. The upconverted signal at $\omega_{F W M}$ is retained by eliminating the signals at $\omega_{c}$ and $\omega_{L O O S}$ through optical filtering. As shown in Fig. 41, optical filter 2 then converts the ODSB signal at $\omega_{F W M}$ to the OSSB signal, which is more chromatic dispersion tolerant. This signal is then transmitted over the fiber and photo-detected in the RAP for generating a RF signal at $\omega_{L} O$. The strength of the FWM signal can be increased by employing the Stimulated Raman Scattering (SRS) described in Section II-B3 [91]. As shown in Fig. 41, the output of a laser, that is referred to as Raman Pump (RP), is counter-propagated, where the energy from the higher-frequency, higher-power pump signal is transferred to the lower-frequency, lower-power FWM with the aid of the SRS phenomenon discussed in Section II-B3 [91]. Although the architecture of Fig. 41 employs a single baseband-data modulated wavelength at $\omega_{c}$, this concept can be extended to a WDM signal that is generated by combining the data modulated outputs of several lasers. This WDM signal would then be combined with the LOOS at $\omega_{\text {LOOS }}$ and employed for optical upconversion [91].

2) Techniques Based on the Non-Linearity of the Semiconductor Optical Amplifiers (SOAs): SOAs exhibit non-linearities 


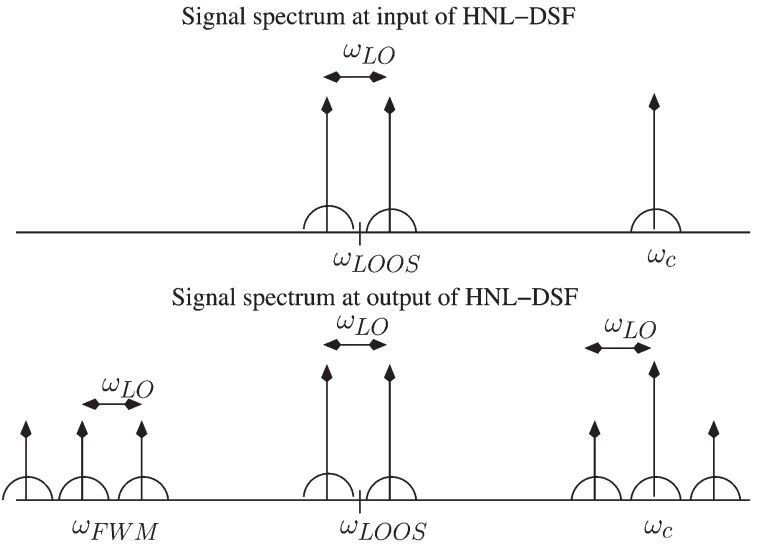

Fig. 42. Four Wave Mixing (FWM) assisted upconversion spectrum.

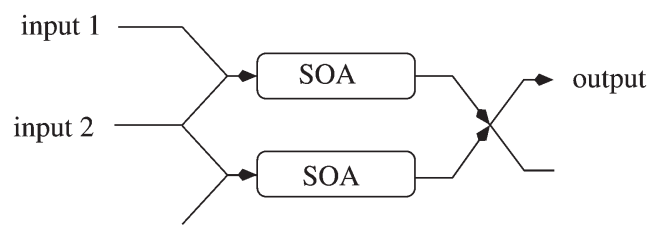

Fig. 43. Semiconductor Optical Amplifier-Mach Zehnder Interferometer (SOA-MZI)

similar to those in optical fibers. Additionally, the SOAs also suffer from gain saturation. The SOAs can be utilised for optical upconversion by exploiting the XPM, FWM and Cross Gain Modulation (XGM) phenomena.

XPM occurs in a SOA in a manner similar to that in fibers, where an architecture similar to that in Fig. 39 can be used, except that the signals at $\omega_{C}$ and $\omega_{L O O S}$ are injected into a SOA-Mach Zehnder Interferometer (SOA-MZI) instead of the NOLM [63] of Fig. 39. Fig. 43 illustrates the architecture of an SOA-MZI, where the combining of the phase modulated signals in the SOA-MZI results in intensity modulation.

Furthermore, the phenomenon of FWM can also be exploited using SOAs, where a LOOS at $\omega_{\text {LOOS }}$ and an optical signal at $\omega_{c}$ carrying the baseband/IF signal are fed to a SOA. This architecture is similar to that of Fig. 41, except that the phenomenon of FWM in SOA is employed instead of the SRSassisted FWM in HNL-DSF, where the process of FWM results in the generation of optical signals at new wavelengths, which result in upconverted RF signals after photo-detection in the RAP [92].

On the other hand, the optical gain of a SOA saturates as the total input power increases. As shown in Fig. 44, a highpower $\mathrm{z} P_{x}(t) \mathrm{dBm}$ optical signal $x_{\omega_{c}}(t)$ at $\omega_{c}$ that carries a baseband/IF signal and a low-power $P_{y}(t) \mathrm{dBm}$ optical LOOS $y_{\omega_{\text {LOOS }}}(t)$ at $\omega_{\text {LOOS }}$ are combined and entered into the SOA. The gain $K\left\{P_{x}(t)+P_{y}(t)\right\}$ of the SOA varies as per the instantaneous power of the high-power optical signal at $\omega_{c}$, i.e., the output $z(t)$ of the amplifier becomes:

$$
\begin{aligned}
z(t) & =K\left\{P_{x}(t)+P_{y}(t)\right\} \cdot\left[x_{\omega_{c}}(t)+y_{\omega_{L O O S}}(t)\right] \\
& =K\left\{P_{x}(t)\right\}\left[x_{\omega_{c}}(t)+y_{\omega_{L O O S}}(t)\right] \text { as } P_{x}(t)+P_{y}(t) \approx P_{x}(t) \\
& =K\left\{P_{x}(t)\right\} \cdot x_{\omega_{c}}(t)+K\left\{P_{x}(t)\right\} \cdot y_{\omega_{L O O S}}(t) .
\end{aligned}
$$

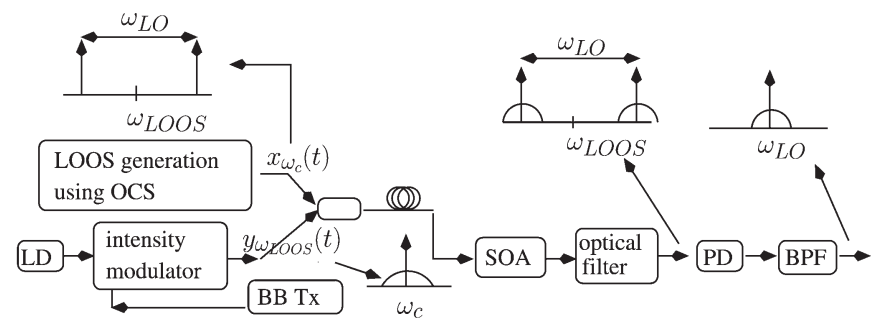

Fig. 44. Cross Gain Modulation (XGM) assisted upconversion in a Semiconductor Optical Amplifier (SOA).

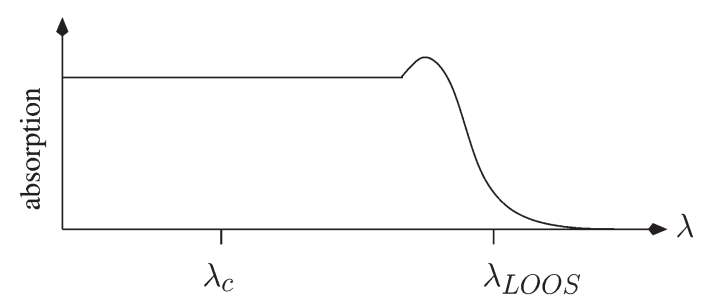

Fig. 45. Electro-Absorption Modulator (EAM) absorption at different optical wavelengths.

Thus, it can be seen from the second term of Equation (32) that the low-power LOOS is modulated in accordance with the intensity of the high-power signal. This effect is referred to as Cross Gain Modulation (XGM) [90]. As shown in Fig. 44, the signal at $\omega_{c}$ is eliminated from the output of the SOA by the optical filter, where the filtered optical spectrum of the XGM-modulated LOOS at $\omega_{\text {LOOS }}$ is shown in Fig. 44. After photodetection in the RAP, the modulated LOOS generates an upconverted RF signal at $\omega_{L O}$, as shown in Fig. 44 [90].

3) Techniques Based on the Non-Linearity of the EAM: Fig. 45 shows the EAM's absorption/transmittance characteristics. While the wavelength $\lambda_{c}$, corresponding to frequency of $f_{c}=\frac{\omega_{c}}{2 \pi} \mathrm{Hz}$, is largely absorbed, the wavelength $\lambda_{L O O S}$, corresponding to frequency of $f_{L O O S}=\frac{\omega_{L O O S}}{2 \pi} \mathrm{Hz}$, is partially transmitted/absorbed. Fig. 46 shows the block diagram of an architecture that relies on the EAM's non-linearity for achieving optical upconversion, where, in the BS, a high power optical signal at $\omega_{c}$ that carries IF/baseband data enters port 1 and exits from port 2 of the optical circulator, which is then injected into an EAM. A LOOS at $\omega_{\text {LOOS }}$ is also injected into the EAM. The absorption of the high power optical signal at $\omega_{c}$ results in the edge of the absorption spectrum ${ }^{9}$ seen in Fig. 45 shifting to lower wavelengths due to the EAM's non-linearity. Hence, as the instantaneous power of the signal at $\omega_{c}$ increases, the extent of absorption of the signal at $\omega_{L O O S}$ drops and vice-versa [64], [93]. Thus, the signal at $\omega_{\text {LOOS }}$ is intensity modulated by the instantaneous power of the signal at $\omega_{c}$, thereby resulting in the modulation of the LOOS at $\omega_{L O O S}$ by the IF/baseband data that was on the optical signal at $\omega_{c}$. This phenomenon is referred to as Cross Absorption Modulation (XAM) [64]. As shown in Fig. 46, the modulated LOOS enters port 2 and exits via port 3 of the optical circulator. It is then transmitted to the RAP and photo-detected for generating an upconverted signal

\footnotetext{
${ }^{9} \mathrm{~A}$ plot representing the extent to which various frequencies are absorbed.
} 


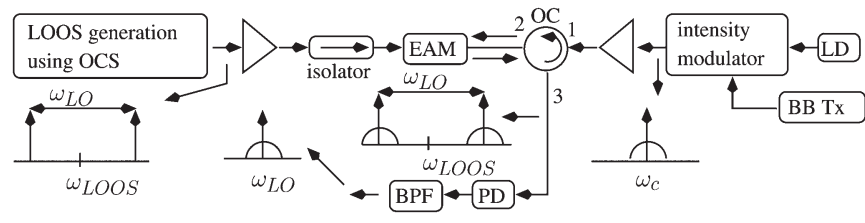

Fig. 46. Optical upconversion using Cross Absorption Modulation (XAM) in a Semiconductor Optical Amplifier (SOA).

TABLE I

COMPARISON OF WAVELENGTH CONVERSION TECHNIQUES

\begin{tabular}{|l|l|}
\hline $\begin{array}{l}\text { phenomenon/hardware } \\
\text { employed }\end{array}$ & challenges \\
\hline fiber-based & large length of fiber needed \\
\hline SOA-based & $\begin{array}{l}\text { low modulation bandwidth, carrier saturation } \\
\text { and polarization sensitivity }\end{array}$ \\
\hline FWM & lower conversion efficiency than XPM/XGM \\
\hline XPM/XGM & $\begin{array}{l}\text { conversion efficiency depends on bit rate } \\
\text { and modulation format }\end{array}$ \\
\hline
\end{tabular}

at $\omega_{L O}$ [64]. An isolator is used in the architecture of Fig. 46 for blocking the propagation of light into the LOOS generation module.

4) Design Guidelines When Using Wavelength Conversion Techniques: The disadvantage of the techniques that exploit fiber non-linearity is the large length of the HNL-DSF that is needed. On the other hand, SOA amplifiers have a low modulation bandwidth and also suffer from a carrier-saturation effect [61]. The limited modulation bandwidth makes it difficult to employ a LOOS whose photo-detection would generate high RF signals, whilst the carrier saturation in a SOA limits the number of WDM channels that may be employed and hence it limits the capacity of the ROF network [61].

The advantage of FWM-based optical upconversion is that FWM does not depend on the bit-rate and on the wireless/optical modulation format [91]. Since XPM and XGM depend on the instantaneous optical power level, the efficiency of the wavelength convertors based on these techniques depends both on the bit-rate and on the optical/wireless modulation formats employed, where only intensity modulated baseband/IF optical signals can be upconverted [61]. The main disadvantage of employing a XGM-based wavelength convertor is the degradation of the extinction-ratio ${ }^{10}$ due to the underlying bitrate versus extinction-ratio trade-off. However, the conversion efficiency of the wavelength convertors based on XPM/XGM is higher than those based on FWM. The discussion within this section has been summarized in Table 1 .

Let us now discuss the final set of techniques conceived for optical upconversion, namely the family of techniques seen in Fig. 22 that rely on the non-linearity of the optical receiver. In other words, they rely on the non-linearity of the photo-detector.

\section{Optical Upconversion Using the Non-Linearity of the Photo-Detector}

The non-linear behaviour of the photo-detector can be exploited for achieving optical upconversion, where the mixing

\footnotetext{
${ }^{10}$ Extinction-ratio is the ratio of the optical power transmitted for bit 1 to that transmitted for bit 0 . It has an ideal value of $\infty$.
}

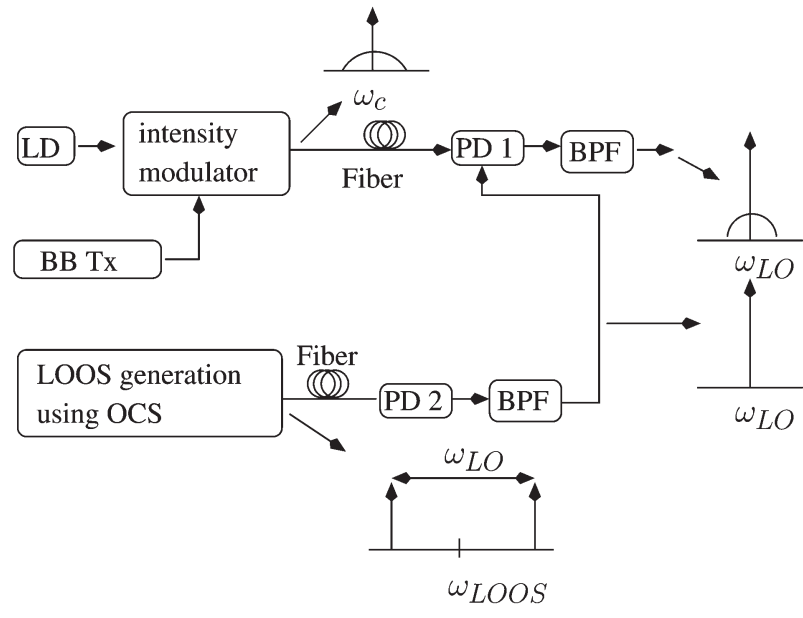

Fig. 47. Optical upconversion by modulating PD bias.

efficiency and hence the strength of the upconverted signal is not high [95]. The architectures that implement this kind of optical upconversion include modulating the bias voltage of the photo-detector as well as exploiting the non-linear nature of the photo-detector responsivity.

A photo-detector consists of a reverse biased diode that generates a current when illuminated with light, where the bias voltage is normally constant. Modulating the bias voltage of the photodetector can be used for optical upconversion[62], [95]. Fig. 47 shows the block diagram of optical upconversion relying on the modulation of the photo-detector's bias voltage, where the baseband/IF modulated optical signal at $\omega_{c}$ and a LOOS at $\omega_{\text {LOOS }}$ are transmitted over two different fiber links from the BS to the RAP. The LOOS is generated at the BS and remotely transmitted to the RAP for photo-detection by PD 2 of Fig. 47 for generating a RF tone at $\omega_{L O}$, which is then applied to the bias terminal of the photo-detector PD 1 employed for detecting the optical signal at $\omega_{c}$. This would result in the photo-detected signal containing upconverted data at $\omega_{R F}=$ $\left(\omega_{L O} \pm \omega_{I F}\right)$ [96], where $f_{I F}=\frac{\omega_{I F}}{2 \pi}$ is the frequency of the baseband/IF data. A single fiber link can also be employed instead of employing two fiber links, provided $\omega_{c}$ and $\omega_{L O O S}$ are not the same. Moreover, in such a scenario, optical filters would be needed at the BS to separate the signals at $\omega_{c}$ and $\omega_{\text {LOOS }}$.

On the other hand, the photo-detector current is ideally linearly dependent on the incident optical power. However, the photo-detector's response becomes strongly non-linear, when the incident optical power is high and the reverse bias voltage is low [95], [97]. Fig. 48 shows the block diagram of optical upconversion relying on the non-linear responsivity of the photo-detector, where, in the BS, the baseband/IF modulated optical signal at $\omega_{c}$ and a LOOS at $\omega_{\operatorname{LOOS}}$ are transmitted over the fiber and are then incident on a nonlinear photo-detector in the RAP [95]. The photo-detected signal contains the upconverted data at $\omega_{R F}=\left(\omega_{L O} \pm \omega_{I F}\right)$, where $f_{I F}=\frac{\omega_{I F}}{2 \pi}$ is the frequency of the baseband/IF data [95]. The disadvantage of this technique is that reducing the reverse-bias voltage decreases the detection bandwidth of the photo-detector. 


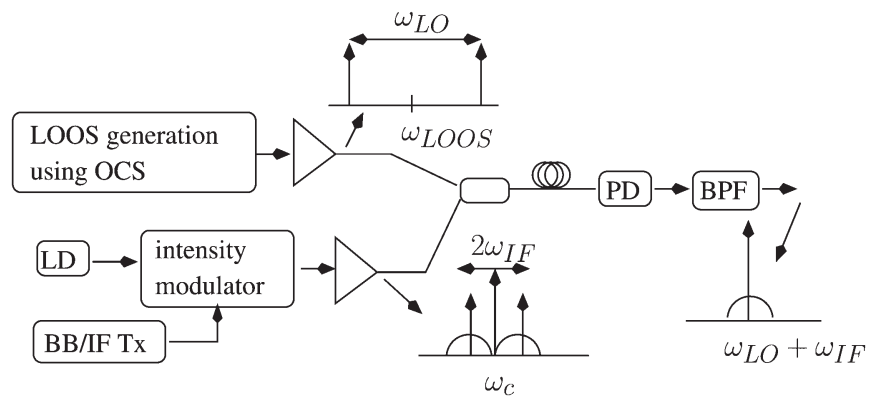

Fig. 48. Optical upconversion using non-linear responsivity.

\section{Conclusion And Design Guidelines}

The Radio Frequency (RF) carriers are becoming overcrowded due to the increased number of wireless communications subscribers. Hence, the cellular operators are considering migration to higher RF carriers. The performance of the wireless link is limited by the performance of the RF mixer at high RF carriers. It is against this backdrop that optical up-conversion helps avoid the employment of high RF mixers. Additionally, minimizing the high-frequency RF front-end in the RAPs reduces the installation cost. In this paper, we introduced the basics of ROF communication followed by a brief portrayal of the advanced optical upconversion techniques that exploit the non-linearity of the ROF link. Optical upconversion architectures that rely on the MZM's non-linearity, that employ wavelength conversion techniques or exploit the photo-detector's non-linearity were discussed in this paper.

Techniques relying on the MZM's non-linearity either exploit the optical harmonics, namely the optical N-tupling techniques or they harness the optical intermodulation products. Optical $\mathrm{N}$-tupling is preferred when the photo-detected RF signal should be at a frequency, which is a multiple of the frequency of the RF signal employed at the transmitter. The design guidelines of the optical N-tupling techniques were presented in Section IV-A2. On the other hand, the techniques relying on the intermodulation products discussed in Section IV-A3 are employed, when a baseband signal has to be upconverted to RF without using any electronic mixer. Using wavelength conversion based non-linear techniques facilitates cost savings by enabling wavelength re-use [90] and these techniques do not rely on the polarization-sensitive MZM. An additional advantage of these techniques is the ability to move data from one wavelength to another, thereby facilitating optical switching [37]. Moreover, using a $f_{\text {LOOS }} \mathrm{Hz}$ wavelength having different values of the $f_{L O} \mathrm{~Hz}$ LOOS facilitates wireless frequency selection in WDM-ROF networks. Wavelength conversion techniques exploit the non-linearity of fibers, of the SOA or of the EAM. Section IV-B4 provides the design guidelines for these techniques. Finally, optical upconversion can also be implemented by modulating the photo-detector's bias voltage or by exploiting the non-linear nature of the photodetector's responsivity. Some areas of potential research are the integration of the various millimeter-wave techniques with a ROF backhaul [98], [99], the employment of mode-division multiplexing in multimode optical fibers for ROF [100], using phased array antennas in a ROF link [22] and the employment of plastic optical fiber in indoor personal area networks [21].
ABBREVIATIONS

AWG Arrayed Waveguide Grating

ASE

BER

BPF

BS

CoMP

DAS

DC

DEMUX

DIPP

EAM

$\mathrm{EO}$

FBG

FM

FWM

GVD

HNL-DSF

IF

IL

IM

LD

LO

Amplified Spontaneous Emission

Bit Error Ratio

Band Pass Filter

Base Station

Co-ordinated Multipoint

Distributed Antenna System

Direct Current

Demultiplexer

Dispersion Induced Power Penalty

Electro-Absorbtion Modulator

Electronic-to-optical

Fiber Bragg Gratings

Frequency Modulation

Four Wave Mixing

Group Velocity Dispersion

LOOS Local Oscillator Optical Signal

MATP Maximum Transmission Point

MIMO Multiple Input Multiple Output

MITP Minimum Transmission Point

MS

MUX

MZI

MZM

NOLM

OADM

OC

OCS

ODSB

$\mathrm{OE}$

Mobile Station

Multiplexer

Mach-Zehnder delay Interferometer

Mach-Zehnder Modulator

OFM

OSSB

PD

PM

QP

RAPS

Non-linear Optical Loop Mirror

RIN

RF

RF Tx Optical Add Drop Multiplexers

Optical Coupler

Optical Carrier Suppression

Optical Double Side Band

Optical-to-electronic

Optical Frequency Multiplication

Optical Single Side Band

Photo-Diode

Phase Modulation

Quadrature Point

Radio Access Points

Relative Intensity Noise

RF Transmitter

ROF Radio Over Fiber

RP Raman Pump

SCM

SBS

SOA

SOA-MZI

SPM

SRS

WDM

XPM

XGM

XAM
Sub-Carrier Multiplexing

Stimulated Brillouin Scattering

Semiconductor Optical Amplifier

SOA-Mach Zehnder Interferometer

Self-Phase Modulation

Stimulated Raman Scattering

Wavelength Division Multiplexing

Cross Phase Modulation

Cross Gain Modulation

Cross Absorption Modulation 


\section{REFERENCES}

[1] V. Thomas, M. El-Hajjar, and L. Hanzo, "Performance improvement and cost reduction techniques for radio over fiber communications," IEEE Commun. Surveys Tuts., to be published.

[2] D. Wake, A. Nkansah, and N. Gomes, "Radio over fiber link design for next generation wireless systems," J. Lightw. Technol., vol. 28, no. 16, pp. 2456-2464, Aug. 2010.

[3] X. Fernando, "Radio over fiber-An optical technique for wireless access," Ryerson Commun. Lab., Toronto, ON, Canada, Oct. 2009, IEEE Communications Society Presentation.

[4] G. H. Nguyen, B. Cabon, and Y. Le Guennec, "Generation of $60-\mathrm{GHz}$ MB-OFDM signal-over-fiber by up-conversion using cascaded external modulators," J. Lightw. Technol., vol. 27, no. 11, pp. 1496-1502, Jun. 2009.

[5] V. Thomas, S. Ghafoor, M. El-Hajjar, and L. Hanzo, "A full-duplex diversity-assisted hybrid analogue/digitized radio over fibre for optical/ wireless integration," IEEE Commun. Lett., vol. 17, no. 2, pp. 1-4, Feb. 2013.

[6] V. A. Thomas, S. Ghafoor, M. El-Hajjar, and L. Hanzo, "Baseband radio over fiber aided millimeter-wave distributed antenna for optical/wireless integration," IEEE Commun. Lett., vol. 17, no. 5, pp. 1012-1015, May 2013.

[7] S. Ghafoor and L. Hanzo, "Sub-carrier-multiplexed duplex 64-QAM radio-over-fiber transmission for distributed antennas," IEEE Commun. Lett., vol. 15, no. 12, pp. 1368-1371, Dec. 2011.

[8] S. Ghafoor, V. A. Thomas, and L. Hanzo, "Duplex digitized transmission of 64-QAM signals over a single fiber using a single pulsed laser source," IEEE Commun. Lett., vol. 16, no. 8, pp. 1312-1315, Aug. 2012.

[9] W. Stephens, T. Joseph, and B. Chen, "Analog microwave fiber optic communications links," in Proc. IEEE MTT-S Int. Microw. Symp. Dig., Jun. 1984, pp. 533-534.

[10] X. Fernando and A. Sesay, "Adaptive asymmetric linearization of radio over fiber links for wireless access," IEEE Trans. Veh. Technol., vol. 51, no. 6, pp. 1576-1586, Nov. 2002.

[11] X. Fernando and A. Sesay, "A Hammerstein-type equalizer for concatenated fiber-wireless uplink," IEEE Trans. Veh. Technol., vol. 54, no. 6, pp. 1980-1991, Nov. 2005.

[12] X. N. Fernando, Radio Over Fiber for Wireless Communications: From Fundamentals to Advanced Topics. Hoboken, NJ, USA: Wiley, 2014.

[13] J. Mitchell, "Optical networks (radio-over-fiber networks)," in Broadband Access Networks. Berlin, Germany: Springer-Verlag, May 2009, pp. $283-300$

[14] S. Karabetsos, S. Mikroulis, and A. Nassiopoulos, "Radio over fiber for broadband communications: A promising technology for next generation networks," in Handbook of Research on Heterogeneous Next Generation Networking: Innovations and Platforms. Hershey, PA, USA: IGI Global, 2008, pp. 80-103.

[15] G. Shen, R. Tucker, and C.-J. Chae, "Fixed mobile convergence architectures for broadband access: Integration of EPON and WiMAX [topics in optical communications]," IEEE Commun. Mag., vol. 45, no. 8, pp. 44-50, Aug. 2007.

[16] N. Ghazisaidi, M. Maier, and C. Assi, "Fiber-wireless (FiWi) access networks: A survey," IEEE Commun. Mag., vol. 47, no. 2, pp. 160-167, Feb. 2009.

[17] R. Llorente et al., "Ultra-wideband radio signals distribution in FTTH networks," IEEE Photon. Technol. Lett., vol. 20, no. 11, pp. 945-947, Jun. 2008.

[18] P. K. Tang, L. C. Ong, A. Alphones, B. Luo, and M. Fujise, "PER and EVM measurements of a radio-over-fiber network for cellular and WLAN system applications," J. Lightw. Technol., vol. 22, no. 11, pp. 2370-2376, Nov. 2004.

[19] W. Way, R. Wolff, and M. Krain, "A 1.3- $\mu \mathrm{m}$ 35-km fiber-optic microwave multicarrier transmission system for satellite earth stations," J. Lightw. Technol., vol. 5, no. 9, pp. 1325-1332, Sep. 1987.

[20] B. Lannoo, D. Colle, M. Pickavet, and P. Demeester, "Radio-over-fiberbased solution to provide broadband internet access to train passengers [topics in optical communications]," IEEE Commun. Mag., vol. 45, no. 2, pp. 56-62, Feb. 2007.

[21] A. Koonen and E. Tangdiongga, "Photonic home area networks," J. Lightw. Technol., vol. 32, no. 4, pp. 591-604, Feb. 2014.

[22] K. Lau, G. Lutes, and R. Tjoelker, "Ultra-stable RF-over-fiber transport in NASA antennas, phased arrays and radars (invited)," J. Lightw. Technol., vol. 32, no. 20, pp. 3440-3451, Oct. 2014.

[23] O. Omomukuyo, M. Thakur, and J. Mitchell, "Simple 60-GHz MBOFDM ultrawideband ROF system based on remote heterodyning," IEEE Photon. Technol. Lett., vol. 25, no. 3, pp. 268-271, Feb. 2013.
[24] K. Kitayama et al., "An approach to single optical component antenna base stations for broad-band millimeter-wave fiber-radio access systems," IEEE Trans. Microw. Theory Tech., vol. 48, no. 12, pp. 25882595, Dec. 2000

[25] J. J. Vegas Olmos, T. Kuri, and K. Kitayama, "Dynamic reconfigurable WDM 60-GHz millimeter-waveband radio-over-fiber access network: Architectural considerations and experiment," J. Lightw. Technol., vol. 25, no. 11, pp. 3374-3380, Nov. 2007.

[26] L. Hanzo, Y. Akhtman, L. Wang, and M. Jiang, MIMO-OFDM for LTE, WiFi and WiMax: Coherent Versus Non-Coherent and Co-Operative Turbo Transceivers, 1st ed. Hoboken, NJ, USA: Wiley, 2011.

[27] L. Hanzo, O. Alamri, M. El-Hajjar, and N. Wu, Near-Capacity MultiFunctional MIMO Systems: Sphere-Packing, Iterative Detection, and Cooperation, 1st ed. Piscataway, NJ, USA: Wiley/IEEE Press, 2009.

[28] L. Hanzo, M. El-Hajjar, and O. Alamri, "Near-capacity wireless transceivers and cooperative communications in the MIMO era: Evolution of standards, waveform design, future perspectives," Proc. IEEE, vol. 99 , no. 8, pp. 1343-1385, Aug. 2011

[29] H. Al-Raweshidy and S. Komaki, Radio Over Fiber Technologies for Mobile Communication Networks, 1st ed. Norwood, MA, USA: Artech House, 2002.

[30] Z. Jia, J. Yu, and G. K. Chang, "A full-duplex radio-over-fiber system based on optical carrier suppression and reuse," IEEE Photon. Technol. Lett., vol. 18, no. 16, pp. 1726-1728, Aug. 2006.

[31] S. Mikroulis, S. Karabetsos, E. Pikasis, and A. Nassiopoulos, "Performance evaluation of a Radio Over Fiber (ROF) system subject to the transmitter's limitations for application in broadband networks," IEEE Trans. Consum. Electron., vol. 54, no. 2, pp. 437-443, May 2008.

[32] J. Ma et al., "Fiber dispersion influence on transmission of the optical millimeter-waves generated using LN-MZM intensity modulation," J. Lightw. Technol., vol. 25, no. 11, pp. 3244-3256, Nov. 2007.

[33] R. Kalman, J. Fan, and L. Kazovsky, "Dynamic range of coherent analog fiber-optic links," J. Lightw. Technol., vol. 12, no. 7, pp. 1263-1277, Jul. 1994.

[34] G. Smith, D. Novak, and Z. Ahmed, "Overcoming chromatic-dispersion effects in fiber-wireless systems incorporating external modulators," IEEE Trans. Microw. Theory Tech., vol. 45, no. 8, pp. 1410-1415, Aug. 1997.

[35] G. L. Li and P. K. Yu, "Optical intensity modulators for digital and analog applications," J. Lightw. Technol., vol. 21, no. 9, pp. 2010-2030, Sep. 2003

[36] K. Ikeda, T. Kuri, and K. Kitayama, "Simultaneous three-band modulation and fiber-optic transmission of $2.5-\mathrm{Gb} / \mathrm{s}$ baseband, microwave, 60-GHz-band signals on a single wavelength," J. Lightw. Technol., vol. 21, no. 12, pp. 3194-3202, Dec. 2003.

[37] G. P. Agrawal, Fiber-Optic Communication Systems, 3rd ed. Hoboken, NJ, USA: Wiley, 2002.

[38] G. P. Agrawal, Nonlinear Fiber Optics, 4th ed. San Diego, CA, USA Academic, 2007.

[39] C. Cox, III, E. Ackerman, R. Helkey, and G. Betts, "Direct-detection analog optical links," IEEE Trans. Microw. Theory Tech., vol. 45, no. 8, pp. 1375-1383, Aug. 1997.

[40] T. Rappaport et al., "Millimeter wave mobile communications for 5G cellular: It will work!" IEEE Access, vol. 1, pp. 335-349, May 2013

[41] W. Roh et al., "Millimeter-wave beamforming as an enabling technology for 5G cellular communications: Theoretical feasibility and prototype results," IEEE Commun. Mag., vol. 52, no. 2, pp. 106-113, Feb. 2014

[42] X. Fernando and A. Anpalagan, "On the design of optical fiber based wireless access systems," in Proc. IEEE ICC, Jun. 2004, vol. 6, pp. 3550-3555.

[43] X. Fernando, "Improved expression for intensity noise in multimedia over fiber networks," in Proc. 1st Int. Conf. Ind. Inf. Syst., Aug. 2006, pp. $425-429$.

[44] G. Smith and D. Novak, "Broad-band millimeter-wave (38 GHz) fiberwireless transmission system using electrical and optical SSB modulation to overcome dispersion effects," IEEE Photon. Technol. Lett., vol. 10, no. 1, pp. 141-143, Jan. 1998.

[45] C. Carlsson, A. Larsson, and A. Alping, "RF transmission over multimode fibers using VCSELs-Comparing standard and high-bandwidth multimode fibers," J. Lightw. Technol., vol. 22, no. 7, pp. 1694-1700, Jul. 2004.

[46] J. Corral, J. Marti, and J. Fuster, "General expressions for IM/DD dispersive analog optical links with external modulation or optical upconversion in a Mach-Zehnder electrooptical modulator," IEEE Trans. Microw. Theory Tech., vol. 49, no. 10, pp. 1968-1976, Oct. 2001. 
[47] J. Park, W. Sorin, and K. Lau, "Elimination of the fibre chromatic dispersion penalty on $1550 \mathrm{~nm}$ millimetre-wave optical transmission," Electron. Lett., vol. 33, no. 6, pp. 512-513, Mar. 1997.

[48] R. Kashyap, Fiber Bragg Gratings, 1st ed. San Diego, CA, USA: Academic, 1999.

[49] J. Yu et al., "DWDM optical millimeter-wave generation for radioover-fiber using an optical phase modulator and an optical interleaver," IEEE Photon. Technol. Lett., vol. 18, no. 13, pp. 1418-1420, Jul. 2006.

[50] G. Smith, D. Novak, and Z. Ahmed, "Technique for optical SSB generation to overcome dispersion penalties in fibre-radio systems," Electron. Lett., vol. 33, no. 1, pp. 74-75, Jan. 1997.

[51] J. O'Reilly, P. Lane, R. Heidemann, and R. Hofstetter, "Optical generation of very narrow linewidth millimetre wave signals," Electron. Lett. vol. 28, no. 25, pp. 2309-2311, Dec. 1992.

[52] M. Weiss et al., "60-GHz photonic millimeter-wave link for short to medium range wireless transmission up to $12.5 \mathrm{~Gb} / \mathrm{s}$," J. Lightw. Technol., vol. 26, no. 15, pp. 2424-2429, Aug. 2008.

[53] V. Urick, F. Bucholtz, P. Devgan, J. McKinney, and K. Williams, "Phase modulation with interferometric detection as an alternative to intensity modulation with direct detection for analog-photonic links," IEEE Trans. Microw. Theory Tech., vol. 55, no. 9, pp. 1978-1985, Sep. 2007.

[54] M. LaGasse and S. Thaniyavaru, "Bias-free high-dynamic-range phasemodulated fiber-optic link," IEEE Photon. Technol. Lett., vol. 9, no. 5, pp. 681-683, May 1997.

[55] R. Olshansky, "Microwave subcarrier multiplexing: New approach to wideband lightwave systems," IEEE Circuits Devices Mag., vol. 4, no. 6, pp. 8-14, Nov. 1988

[56] H. Toda, T. Yamashita, T. Kuri, and K. Kitayama, "Demultiplexing using an arrayed-waveguide grating for frequency-interleaved DWDM millimeter-wave radio-on-fiber systems," J. Lightw. Technol., vol. 21, no. 8, pp. 1735-1741, Aug. 2003.

[57] C. Oh and W. Gu, "Fiber induced distortion in a subcarrier multiplexed lightwave system," IEEE J. Sel. Areas Commun., vol. 8, no. 7, pp. 12961303, Sep. 1990.

[58] R. Hui et al., "Subcarrier multiplexing for high-speed optical transmission," J. Lightw. Technol., vol. 20, no. 3, pp. 417-427, Mar. 2002

[59] R. Gross and R. Olshansky, "Multichannel coherent FSK experiments using subcarrier multiplexing techniques," J. Lightw. Technol., vol. 8, no. 3, pp. 406-415, Mar. 1990.

[60] M. Smit and C. Van Dam, "PHASAR-based WDM-devices: Principles, design and applications," IEEE J. Sel. Topics Quantum Electron., vol. 2, no. 2, pp. 236-250, Jun. 1996.

[61] Z. Jia, J. Yu, and G. K. Chang, "All-optical $16 \times 2.5 \mathrm{~Gb} / \mathrm{s}$ WDM signal simultaneous up-conversion based on XPM in an NOLM in ROF systems," IEEE Photon. Technol. Lett., vol. 17, no. 12, pp. 2724-2726, Dec. 2005.

[62] C. S. Choi, H.-S. Kang, W.-Y. Choi, D.-H. Kim, and K.-S. Seo, "Phototransistors based on InP HEMTs and their applications to millimeterwave radio-on-fiber systems," IEEE Trans. Microw. Theory Tech., vol. 53, no. 1, pp. 256-263, Jan. 2005.

[63] H. J. Song, J. S. Lee, and J.-I. Song, "Error-free simultaneous all-optical upconversion of WDM radio-over-fiber signals," IEEE Photon. Technol. Lett., vol. 17, no. 8, pp. 1731-1733, Aug. 2005.

[64] C. S. Park, C. K. Oh, C. G. Lee, D.-H. Kim, and C.-S. Park, "A photonic up-converter for a WDM radio-over-fiber system using cross-absorption modulation in an EAM," IEEE Photon. Technol. Lett., vol. 17, no. 9, pp. 1950-1952, Sep. 2005.

[65] J. Yu et al., "Optical millimeter-wave generation or up-conversion using external modulators," IEEE Photon. Technol. Lett., vol. 18, no. 1, pp. 265-267, Jan. 2006.

[66] S. J. B. Yoo, "Wavelength conversion technologies for WDM network applications," J. Lightw. Technol., vol. 14, no. 6, pp. 955-966, Jun. 1996.

[67] C.-T. Lin, J. Chen, P.-T. Shih, W.-J. Jiang, and S. Chi, "Ultra-high data-rate $60 \mathrm{GHz}$ radio-over-fiber systems employing optical frequency multiplication and OFDM formats," J. Lightw. Technol., vol. 28, no. 16, pp. 2296-2306, Aug. 2010.

[68] J. Fuster, J. Marti, J. Corral, V. Polo, and F. Ramos, "Generalized study of dispersion-induced power penalty mitigation techniques in millimeterwave fiber-optic links," J. Lightw. Technol., vol. 18, no. 7, pp. 933-940, Jul. 2000.

[69] A. Koonen and L. Garcia, "Radio-over-MMF techniques-Part II: Microwave to millimeter-wave systems," J. Lightw. Technol., vol. 26, no. 15 , pp. $2396-2408$, Aug. 2008.
[70] G. Qi, J. Yao, J. Seregelyi, S. Paquet, and C. Belisle, "Generation and distribution of a wide-band continuously tunable millimeter-wave signal with an optical external modulation technique," IEEE Trans. Microw. Theory Tech., vol. 53, no. 10, pp. 3090-3097, Oct. 2005.

[71] E. Ackerman et al., "Signal-to-noise performance of two analog photonic links using different noise reduction techniques," in Proc. IEEE/MTT-S Int. Microw. Symp., Jun. 2007, pp. 51-54.

[72] C.-T. Lin et al., "Hybrid optical access network integrating fiber-tothe-home and radio-over-fiber systems," IEEE Photon. Technol. Lett., vol. 19, no. 8, pp. 610-612, Apr. 2007.

[73] M. F. Huang, J. Yu, Z. Jia, and G. K. Chang, "Simultaneous generation of centralized lightwaves and double/single sideband optical millimeterwave requiring only low-frequency local oscillator signals for radioover-fiber systems," J. Lightw. Technol., vol. 26, no. 15, pp. 2653-2662, Aug. 2008.

[74] L. Chen, H. Wen, and S. Wen, "A radio-over-fiber system with a novel scheme for millimeter-wave generation and wavelength reuse for up-link connection," IEEE Photon. Technol. Lett., vol. 18, no. 19, pp. 20562058, Oct. 2006.

[75] Z. Jia et al., "Multiband signal generation and dispersion-tolerant transmission based on photonic frequency tripling technology for $60-\mathrm{GHz}$ radio-over-fiber systems," IEEE Photon. Technol. Lett., vol. 20, no. 17, pp. 1470-1472, Sep. 2008.

[76] J. Yu, Z. Jia, T. Wang, and G. K. Chang, "Centralized lightwave radio-over-fiber system with photonic frequency quadrupling for highfrequency millimeter-wave generation," IEEE Photon. Technol. Lett., vol. 19, no. 19, pp. 1499-1501, Oct. 2007.

[77] J. O'Reilly and P. Lane, "Fibre-supported optical generation and delivery of $60 \mathrm{GHz}$ signals," Electron. Lett., vol. 30, no. 16, pp. 1329-1330, Aug. 1994.

[78] C.-T. Lin et al., "Optical millimeter-wave signal generation using frequency quadrupling technique and no optical filtering," IEEE Photon. Technol. Lett., vol. 20, no. 12, pp. 1027-1029, Jun. 2008.

[79] M. Larrode, A. Koonen, J. Olmos, and A. Ng'Oma, "Bidirectional radio-over-fiber link employing optical frequency multiplication," IEEE Photon. Technol. Lett., vol. 18, no. 1, pp. 241-243, Jan. 2006.

[80] P.-T. Shih et al., "Optical millimeter-wave signal generation via frequency 12-tupling," J. Lightw. Technol., vol. 28, no. 1, pp. 71-78, Jan. 2010.

[81] J. Fuster, J. Marti, and J. Corral, "Chromatic dispersion effects in electrooptical upconverted millimetre-wave fibre optic links," Electron. Lett., vol. 33, no. 23, pp. 1969-1970, Nov. 1997.

[82] J. Zhang, H. Chen, M. Chen, T. Wang, and S. Xie, "A photonic microwave frequency quadrupler using two cascaded intensity modulators with repetitious optical carrier suppression," IEEE Photon. Technol. Lett., vol. 19, no. 14, pp. 1057-1059, Jul. 2007.

[83] Q. Chang, H. Fu, and Y. Su, "Simultaneous generation and transmission of downstream multiband signals and upstream data in a bidirectional radio-over-fiber system," IEEE Photon. Technol. Lett., vol. 20, no. 3, pp. 181-183, Feb. 2008

[84] H. Chi and J. Yao, "Frequency quadrupling and upconversion in a radio over fiber link," J. Lightw. Technol., vol. 26, no. 15, pp. 2706-2711, Aug. 2008.

[85] K. Kojucharow et al., "Simultaneous electrooptical upconversion, remote oscillator generation, air transmission of multiple optical WDM channels for a 60-GHz high-capacity indoor system," IEEE Trans. Microw. Theory Tech., vol. 47, no. 12, pp. 2249-2256, Dec. 1999.

[86] M. Larrode, A. Koonen, and J. Olmos, "Fiber-based broadband wireless access employing optical frequency multiplication," IEEE J. Sel. Topics Quantum Electron., vol. 12, no. 4, pp. 875-881, Jul. 2006.

[87] M. Abramowitz and I. A. Stegun, Abramowitz and Stegun: Handbook of Mathematical Functions, 10th ed. Gaithersburg, MD, USA: National Bureau of Standards, 1972, ser. National Bureau of Standards Applied Mathematics Series-55.

[88] W. J. Jiang et al., "Simple 14-Gb/s short-range radio-over-fiber system employing a single-electrode MZM for 60-GHz wireless applications," J. Lightw. Technol., vol. 28, no. 16, pp. 2238-2246, Aug. 2010.

[89] Y. Le Guennec and R. Gary, "Optical frequency conversion for millimeter-wave ultra-wideband-over-fiber systems," IEEE Photon. Technol. Lett., vol. 19, no. 13, pp. 996-998, Jul. 2007.

[90] J. H. Seo et al., "SOA-EAM frequency up/down-converters for $60-\mathrm{GHz}$ bi-directional radio-on-fiber systems," IEEE Trans. Microw. Theory Tech., vol. 54, no. 2, pp. 959-966, Feb. 2006.

[91] J. Yu, J. Gu, X. Liu, Z. Jia, and G. K. Chang, "Seamless integration of an $8 \times 2.5 \mathrm{~Gb} / \mathrm{s}$ WDM-PON and radio-over-fiber using all-optical up-conversion based on Raman-assisted FWM," IEEE Photon. Technol. Lett., vol. 17, no. 9, pp. 1986-1988, Sep. 2005. 
[92] H. Kim et al., "An all-optical frequency up-converter utilizing four-wave mixing in a semiconductor optical amplifier for sub-carrier multiplexed radio-over-fiber applications," Opt. Exp., vol. 15, no. 6, pp. 3384-3389, Mar. 2007.

[93] J. M. Senior, Optical Fiber Communications Principles and Practice, 3rd ed. Upper Saddle River, NJ, USA: Prentice-Hall, 2009.

[94] C. Joergensen et al., "All-optical wavelength conversion at bit rates above $10 \mathrm{~Gb} / \mathrm{s}$ using semiconductor optical amplifiers," IEEE J. Sel. Topics Quantum Electron., vol. 3, no. 5, pp. 1168-1180, Oct. 1997.

[95] M. Tsuchiya and T. Hoshida, "Nonlinear photodetection scheme and its system applications to fiber-optic millimeter-wave wireless downlinks," IEEE Trans. Microw. Theory Tech., vol. 47, no. 7, pp. 1342-1350, Jul. 1999.

[96] H. Ogawa and Y. Kamiya, "Fiber-optic microwave transmission using harmonic laser mixing, optoelectronic mixing, optically pumped mixing," IEEE Trans. Microw. Theory Tech., vol. 39, no. 12, pp. 2045-2051, Dec. 1991.

[97] K. Williams, R. Esman, and M. Dagenais, "Nonlinearities in p-i-n microwave photodetectors," J. Lightw. Technol., vol. 14, no. 1, pp. 84-96, Jan. 1996.

[98] H.-T. Huang, C.-T. Lin, Y.-T. Chiang, C.-C. Wei, and C.-H. Ho, "Simple $2 \times 2$ MIMO 60-GHz optical/wireless system with extending fiber transmission distance," J. Lightw. Technol., vol. 32, no. 20, pp. 36603667, Oct. 2014

[99] S. Mikroulis, O. Omomukuyo, M. Thakur, and J. Mitchell, "Investigation of a SMF-MMF link for a remote heterodyne 60-GHz OFDM ROF based gigabit wireless access topology," J. Lightw. Technol., vol. 32, no. 20, pp. 3645-3653, Oct. 2014.

[100] G. Gordon, M. Crisp, R. Penty, T. Wilkinson, and I. White, "Feasibility demonstration of a mode-division multiplexed MIMO-enabled radioover-fiber distributed antenna system," J. Lightw. Technol., vol. 32, no. 20 , pp. 3521-3528, Oct. 2014.

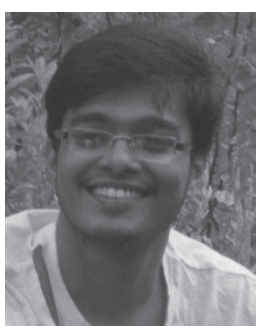

Varghese Antony Thomas received the B.E.(Hons.) degree in electrical and electronics engineering from Birla Institute of Technology and Science, Pilani, India, in 2010, and the M.Sc. degree in wireless communications from the University of Southampton, Southampton, U.K., in 2011. He is currently working toward the $\mathrm{Ph} . \mathrm{D}$. degree with the University of Southampton. His research interests are mainly in optical communications, optical-wireless integration, backhaul for MIMO, and radio over fiber systems. He is a recipient of the several academic awards, including the Commonwealth Scholarship of the Government of U.K. and Mayflower Scholarship of the University of Southampton.

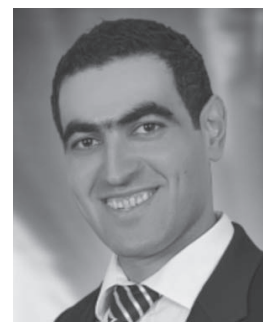

Mohammed El-Hajjar received the B.Eng. degree in electrical engineering from the American University of Beirut, Beirut, Lebanon, in 2004 and the M.Sc. degree in radio frequency communication systems and the Ph.D. degree in wireless communications from the University of Southampton, Southampton, U.K., in 2005 and 2008, respectively. Following his Ph.D. degree, he joined Imagination Technologies as a Design Engineer, where he worked on designing and developing Imagination's multistandard communications platform, which resulted in three patents. In January 2012, he joined the School of Electronics and Computer Science, University of Southampton, as a Lecturer in the Southampton Wireless Research Group. He has published a Wiley-IEEE book and in excess of 50 journal and international conference papers. His research interests are mainly in the development of intelligent communications systems, including energy-efficient transceiver design, cross-layer optimization for large-scale networks, MIMO, millimeter-wave communications, and radio-over-fiber systems. Dr. El-Hajjar was a recipient of several academic awards, including the Dorothy Hodgkin Postgraduate Award and the IEEE ICC2010 Best Paper Award.

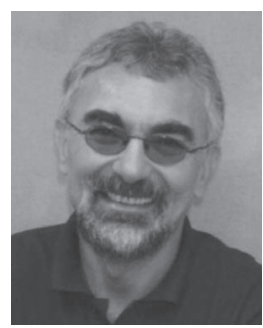

Lajos Hanzo (M'91-SM'92-F'04) received the M.S degree in electronics and the Ph.D. degree from the Technical University of Budapest, Budapest, Hungary, in 1976 and 1983, respectively; the D.Sc. degree from the University of Southampton, Southampton, U.K., in 2004; and the "Doctor Honoris Causa" degree from the Technical University of Budapest in 2009. During his 38-year career in telecommunications, he has held various research and academic posts in Hungary, Germany, and the U.K. Since 1986, he has been with the School of Electronics and Computer Science, University of Southampton, where he holds the Chair in Telecommunications. He is currently directing an academic research team, working on a range of research projects in the field of wireless multimedia communications sponsored by industry, the Engineering and Physical Sciences Research Council U.K., the European IST Programme, and the Mobile Virtual Centre of Excellence, UK. During 2008-2012, he was a Chaired Professor at Tsinghua University, Beijing. He is an enthusiastic supporter of industrial and academic liaison and offers a range of industrial courses. He has successfully supervised about 100 Ph.D. students, coauthored 20 John Wiley/IEEE Press books on mobile radio communications totaling in excess of 10000 pages, and published more than 1400 research entries at IEEE Xplore. Dr. Hanzo is a Fellow of the Royal Academy of Engineering, the Institution of Engineering and Technology, and the European Association for Signal Processing. He is also a Governor of the IEEE Vehicular Technology Society. During 2008-2012, he was the Editor-in-Chief of IEEE Press. He has served as the Technical Program Committee Chair and the General Chair of IEEE conferences, has presented keynote lectures, and has been awarded a number of distinctions. For further information on research in progress and associated publications, please refer to http://www-mobile.ecs.soton.ac.uk. 